\title{
12 Non-Homogeneous Boundary Value Problems: Green's Functions
}

\subsection{Ordinary Differential Equations}

\subsubsection{Definition of a Green's Function}

Suppose that we wish to solve the non-homogeneous DE

$$
\mathfrak{L} u(x) \equiv \frac{d}{d x}\left(p(x) \frac{d u}{d x}\right)-q(x) u(x)=f(x)
$$

in the interval $a \leq x \leq b$ with $f(x)$ a known function and with non-homogeneous boundary conditions

$$
\begin{aligned}
& \alpha_{1} u(a)+\left.\alpha_{2} \frac{d u}{d x}\right|_{x=a}=\alpha_{3} \\
& \beta_{1} u(b)+\left.\beta_{2} \frac{d u}{d x}\right|_{x=b}=\beta_{3}
\end{aligned}
$$

for given values of the constants $\alpha_{1}, \alpha_{2}, \alpha_{3}, \beta_{1}, \beta_{2}$, and $\beta_{3}$.

We recognize that $\mathfrak{L}$ is the Sturm Liouville differential operator and so we know that it satisfies the generalized green's identity. This means that if $u(x)$ and $v(x)$ are any twice- differentiable functions defined on $a \leq x \leq b$,

$$
\int_{a}^{b}[v(x) \mathfrak{L} u(x)-u(x) \mathfrak{L} v(x)] d x=\left[p(x)\left(v(x) \frac{d u}{d x}-u(x) \frac{d v}{d x}\right)\right]_{x=a}^{x=b} .
$$

Let us apply this to functions of our choosing. First we choose $u(x)$ to be the solution of our non-homogeneous boundary value problem. Then, we choose $v(x)$ to be the function $G\left(x ; x^{\prime}\right)$ where

$$
\mathfrak{L} G\left(x ; x^{\prime}\right)=\delta\left(x-x^{\prime}\right) .
$$

With these choices, the identity (12.1.3) gives us

$$
\int_{a}^{b}\left[G\left(x ; x^{\prime}\right) f(x)-u(x) \delta\left(x-x^{\prime}\right)\right] d x=\left[p(x)\left(G\left(x ; x^{\prime}\right) \frac{d u}{d x}-u(x) \frac{d G}{d x}\right)\right]_{x=a}^{x=b} .
$$

The second term on the left hand side is just $-u\left(x^{\prime}\right)$. Therefore, we are going to interchange $x$ and $x^{\prime}, x \leftrightarrow x^{\prime}$, and then rearrange all the terms to yield an expression for $u(x)$ :

$$
u(x)=\int_{a}^{b} G\left(x^{\prime} ; x\right) f\left(x^{\prime}\right) d x^{\prime}-\left[p\left(x^{\prime}\right)\left(G\left(x^{\prime} ; x\right) \frac{d}{d x^{\prime}} u\left(x^{\prime}\right)-u\left(x^{\prime}\right) \frac{d}{d x^{\prime}} G\left(x^{\prime} ; x\right)\right)\right]_{x^{\prime}=a}^{x^{\prime}=b}
$$


This is a very auspicious result for it tells us that if we can determine $G\left(x ; x^{\prime}\right)$, we can simply write down the solution of any other non-homogeneous problem involving $\mathfrak{L}$. The determination of $G\left(x ; x^{\prime}\right)$ begins with the specification of boundary conditions to complement our knowledge of its differential equation. The "surface term" in (12.1.5) tells us that we must impose the homogeneous counterparts to the boundary conditions imposed on $u(x)$ since otherwise we will be unable to make a full evaluation of that term. For example, if the boundary conditions on $u(x)$ are $u(a)=\alpha$ and $u(b)=\beta$, we have to require $G\left(a ; x^{\prime}\right)=0$ and $G\left(b ; x^{\prime}\right)=0$ to eliminate the unknown quantities $\left.\frac{d u}{d x}\right|_{x=a}$ and $\left.\frac{d u}{d x}\right|_{x=b}$. Thus, the Green's function $G\left(x ; x^{\prime}\right)$ is the unique solution of

$$
\mathfrak{L} G\left(x ; x^{\prime}\right)=\delta\left(x-x^{\prime}\right)
$$

subject to

$$
\begin{aligned}
& \alpha_{1} G\left(a ; x^{\prime}\right)+\left.\alpha_{2} \frac{d G}{d x}\right|_{x=a}=0 \\
& \beta_{1} G\left(b ; x^{\prime}\right)+\left.\beta_{2} \frac{d G}{d x}\right|_{x=b}=0 .
\end{aligned}
$$

\subsubsection{Direct Construction of the Sturm Liouville Green's Function}

The Green's function DE (12.1.7) is as close to a homogeneous equation as a non- homogeneous one can be. In fact, $G\left(x ; x^{\prime}\right)$ satisfies $\mathfrak{L} u(x)=0$ everywhere except the point $x=x^{\prime}$. Therefore, we should feel reasonably optimistic about our capacity to solve it. We start by noting some basic attributes of the solution that flow from the properties of the DE and the boundary conditions.

Applying the generalized Green's identity (12.1.3) to $u(x)=G\left(x ; x^{\prime}\right)$ and $v(x)=$ $G\left(x ; x^{\prime \prime}\right)$ we find

$$
G\left(x^{\prime} ; x^{\prime \prime}\right)-G\left(x^{\prime \prime} ; x^{\prime}\right)=\left[p(x)\left(G\left(x ; x^{\prime \prime}\right) \frac{d}{d x} G\left(x ; x^{\prime}\right)-G\left(x ; x^{\prime}\right) \frac{d}{d x} G\left(x ; x^{\prime \prime}\right)\right)\right]_{x=a}^{x=b}=0
$$

because $G$ satisfies homogeneous boundary conditions. Thus, we conclude that $G\left(x ; x^{\prime}\right)$ is symmetric under $x \leftrightarrow x^{\prime}$ :

$$
G\left(x ; x^{\prime}\right)=G\left(x^{\prime} ; x\right) .
$$

As we will see again in Section 12.3.4, the boundary conditions are replaced by an initial condition

$$
G\left(t ; t^{\prime}\right)=0 \text { for } t<t^{\prime}
$$

when the independent variable is time $t,-\infty<t<\infty$. This condition gives expression to the causality principle: $G$ is the response of a system to an instantaneous disturbance at $t=t^{\prime}$ and that response cannot precede its cause. Used in an analogous application of the Green's identity, it changes the symmetry property (12.1.8) from $G\left(x ; x^{\prime}\right)=G\left(x^{\prime} ; x\right)$ to $G\left(t ; t^{\prime}\right)=G\left(-t^{\prime} ;-t\right)$. 
Next, we note that integrating the DE from $x^{\prime}-\varepsilon$ to $x^{\prime}+\varepsilon$ gives us

$$
\left.p(x) \frac{d}{d x} G\left(x ; x^{\prime}\right)\right|_{x=x^{\prime}-\varepsilon} ^{x=x^{\prime}+\varepsilon}-\int_{x^{\prime}-\varepsilon}^{x^{\prime}+\varepsilon} q(x) G\left(x ; x^{\prime}\right) d x=1 .
$$

Equation (12.1.8) tells us that $G\left(x ; x^{\prime}\right)$ is symmetric about $x=x^{\prime}$,

$$
G\left(x^{\prime}+\varepsilon ; x^{\prime}\right)=G\left(x^{\prime} ; x^{\prime}+\varepsilon\right)=G\left(x^{\prime}-\varepsilon ; x^{\prime}\right),
$$

and therefore that it is continuous there. So is $q(x)$. This means that when we take the limit as $\varepsilon \rightarrow 0$, we will have

$$
\lim _{\varepsilon \rightarrow 0} \int_{x^{\prime}-\varepsilon}^{x^{\prime}+\varepsilon} q(x) G\left(x ; x^{\prime}\right) d x=0 .
$$

Thus, while $G\left(x ; x^{\prime}\right)$ is continuous at $x=x^{\prime}$, its first derivative must have a discontinuity there:

$$
\left.\lim _{\varepsilon \rightarrow 0} \frac{d}{d x} G\left(x ; x^{\prime}\right)\right|_{x=x^{\prime}-\varepsilon} ^{x=x^{\prime}+\varepsilon}=\frac{1}{p\left(x^{\prime}\right)}
$$

We shall now use (12.1.8) and (12.1.9) to construct $G\left(x ; x^{\prime}\right)$ from non-trivial solutions of the homogeneous $\mathrm{DE} \mathfrak{L} u(x)=0$.

Let $u<(x)$ be such a solution that satisfies one additional constraint, the homogeneous boundary condition at $x=a$,

$$
\alpha_{1} u<(a)+\left.\alpha_{2} \frac{d u_{<}}{d x}\right|_{x=a}=0
$$

Since we also have

$$
\alpha_{1} G\left(a ; x^{\prime}\right)+\left.\alpha_{2} \frac{d G}{d x}\right|_{x=a}=0
$$

and since these two algebraic equations have to admit a non-trivial solution for at least one of $\alpha_{1}$ and $\alpha_{2}$, the determinant of their coefficients must vanish. In other words, we require

$$
\left.u_{<}(a) \frac{d g\left(x ; x^{\prime}\right)}{d x}\right|_{x=a}-\left.G\left(a ; x^{\prime}\right) \frac{d u_{<}(x)}{d x}\right|_{x=a}=0 .
$$

But this is just the Wronskian of two solutions of the same DE and since it vanishes at a particular point $(x=a)$, it must be identically zero on $a \leq x<x^{\prime}$ and the two solutions must be linearly dependent there. Therefore, we can write

$$
G\left(x ; x^{\prime}\right)=c<u<(x) \text { for } a \leq x<x^{\prime}
$$

where $c_{<}$is some constant. 
Similarly, if $u_{>}(x)$ is a non-trivial solution of $\mathfrak{L} u(x)=0$ that satisfies the homogeneous boundary condition

$$
\beta_{1} u_{>}(b)+\left.\beta_{2} \frac{d u_{>}}{d x}\right|_{x=b}=0,
$$

then

$$
G\left(x ; x^{\prime}\right)=c>u_{>}(x) \text { for } x^{\prime}<x \leq b
$$

where $c>$ is some other constant.

Now we shall impose the continuity of $G\left(x ; x^{\prime}\right)$ and the discontinuity of $\frac{d}{d x} G\left(x ; x^{\prime}\right)$ at $x=x^{\prime}$ :

$$
c_{<} u_{<}\left(x^{\prime}\right)=c_{>} u_{>}\left(x^{\prime}\right)
$$

and

$$
c<\left.\frac{d u<}{d x}\right|_{x=x^{\prime}}-c>\left.\frac{d u>}{d x}\right|_{x=x^{\prime}}=-\frac{1}{p\left(x^{\prime}\right)} .
$$

Solving for $c_{<}$and $c_{>}$, we find

$$
c_{<}=\frac{u_{>}\left(x^{\prime}\right)}{p\left(x^{\prime}\right) W\left(x^{\prime}\right)} \text { and } c_{>}=\frac{u_{<}\left(x^{\prime}\right)}{p\left(x^{\prime}\right) W\left(x^{\prime}\right)}
$$

where $W(x)=u_{<}(x) \frac{d u_{>}}{d x}-u_{>}(x) \frac{d u_{<}}{d x}$ is the Wronskian of $u_{<}(x)$ and $u_{>}(x)$. Thus, our final expression for the Green's function is

$$
G\left(x ; x^{\prime}\right)=\left\{\begin{array}{l}
\frac{u<(x) u>\left(x^{\prime}\right)}{p\left(x^{\prime}\right) W\left(x^{\prime}\right)} \text { for } a \leq x<x^{\prime} \\
\frac{u<\left(x^{\prime}\right) u>(x)}{p\left(x^{\prime}\right) W\left(x^{\prime}\right)} \text { for } x^{\prime}<x \leq b
\end{array}\right.
$$

This is called the direct construction formula for determining $G\left(x ; x^{\prime}\right)$ and it results in a closed form expression that contains no summation or integration to perform. The function $p\left(x^{\prime}\right) W\left(x^{\prime}\right)$ in the denominator is in fact a constant as can be seen from equation (9.2.6)

$$
W(x)=W\left(x_{0}\right) \exp \left\{-\int_{x_{0}}^{x} a(\xi) d \xi\right\}
$$

for the Wronskian of the DE

$$
\frac{d^{2} u}{d x^{2}}+a(x) \frac{d u}{d x}+b(x) u(x)=0
$$

Converting

$$
\frac{d}{d x}\left(p(x) \frac{d u}{d x}\right)-q(x) u(x)=0
$$

into this other canonical form, we see that

$$
a(\xi)=\frac{1}{p(\xi)} \frac{d p}{d \xi}
$$


and so,

$$
W(x)=W\left(x_{0}\right) \frac{p\left(x_{0}\right)}{p(x)} \text { or } p(x) W(x)=\text { a constant. }
$$

Equation (12.1.12) provides a simple prescription for determining Sturm Liouville Green's functions and thence for determining the solutions of non-homogeneous Sturm Liouville problems. The latter, obtained by inserting (12.1.12) into (12.1.5), have a form reminiscent of what we found in Chapter 9 from application of the variation of constants approach to solving non-homogeneous ODE's. However, the present approach represents a major advance on variation of constants because it

- incorporates boundary conditions, and

- can be generalized to apply to PDE’s in any number of dimensions.

There is a possible complication that can arise: the Wronskian that appears in the denominator of (12.1.12) could be zero leaving our Green's function undefined. However, the lack of mathematical definition does not prevent interpretation. If the Wronskian of $u_{<}(x)$ and $u_{>}(x)$ is zero, the two functions have to be proportional to each other and so there must exist a single solution of the homogeneous DE that satisfies both boundary conditions. Since the function $q(x)$ can be written $q(x)=r(x)-\lambda \rho(x)$ for some fixed $\lambda$ and $\rho(x) \geq 0$, the homogeneous DE can be converted into the eigenvalue equation

$$
\frac{d}{d x}\left(p(x) \frac{d u}{d x}\right)-r(x) u(x)=-\lambda \rho(x) u(x)
$$

and we see that the vanishing of the Wronskian implies that $\lambda$ is an eigenvalue of $\mathfrak{L} \equiv \frac{d}{d x}\left(p(x) \frac{d}{d x}\right)-r(x)$. As we will see in the following example, this in turn implies a physical interpretation in terms of resonant behaviour.

\subsubsection{Application: The Bowed Stretched String}

Suppose that we have a stretched string that is subjected to a transverse bowing force. If the force per unit length at position $x$ and time $t$ is $F(x, t)$, the transverse displacement of the string is a solution of the non-homogeneous wave equation

$$
\frac{\partial^{2} \psi}{\partial x^{2}}-\frac{1}{c^{2}} \frac{\partial^{2} \psi}{\partial t^{2}}=-\frac{F(x, t)}{T}
$$

where $T$ is the tension in the string. Suppose further that the bowing force is harmonic so that $F(x, t)=-T f(x) e^{-i \omega t}$. The forced response of the string will then be $\psi(x, t)=$ $u(x) e^{-i \omega t}$ where

$$
\frac{d^{2} u}{d x^{2}}+k^{2} u(x)=f(x), \quad k=\frac{\omega}{c} .
$$

If, as we usually do, require the ends of the string to be fixed, this non-homogeneous ODE will be accompanied by the boundary conditions $u(0)=u(L)=0$. 
The Green's function that we need for this problem is the solution of

$$
\frac{d^{2}}{d x^{2}} G\left(x ; x^{\prime}\right)+k^{2} G\left(x ; x^{\prime}\right)=\delta\left(x-x^{\prime}\right)
$$

subject to $G\left(0 ; x^{\prime}\right)=G\left(L ; x^{\prime}\right)=0$. To apply the direct construction formula we require a solution $u_{<}(x)$ of

$$
\frac{d^{2} u}{d x^{2}}+k^{2} u(x)=0
$$

that satisfies $u_{<}(0)=0$. The general solution of this equation is $u(x)=\left\{\begin{array}{c}\cos k x \\ \sin k x\end{array}\right\}$ and so a solution that meets our boundary condition is

$$
u_{<}(x)=\sin k x, \quad 0 \leq x .
$$

A solution $u_{>}(x)$ that clearly meets the condition at the other boundary, namely $u_{>}(L)=$ 0 , is

$$
u_{>}(x)=\sin k(L-x), \quad x \leq L .
$$

Calculating their Wronskian, we have

$$
\begin{aligned}
W(x) & =u_{<}(x) \frac{d u_{>}}{d x}-\frac{d u_{>}}{d x} u_{>}(x)=-\sin k x k \cos k(L-x)-k \cos k x \sin k(L-x) \\
& =-k \sin k(x+L-x)=-k \sin k L .
\end{aligned}
$$

Therefore, since $p(x) \equiv 1$ in this case, the desired Green's function is

$$
G\left(x ; x^{\prime}\right)= \begin{cases}-\frac{\sin k x \sin k\left(L-x^{\prime}\right)}{k \sin k L} & \text { for } 0 \leq x<x^{\prime} \\ -\frac{\sin k x^{\prime} \sin k(L-x)}{k \sin k L} & \text { for } x^{\prime}<x \leq L\end{cases}
$$

Thus, the solution to our bowed string problem is $\psi(x, t)=u(x) e^{-i \omega t}$ where

$$
\begin{aligned}
u(x) & =\int_{0}^{L} G\left(x ; x^{\prime}\right) f\left(x^{\prime}\right) d x^{\prime} \\
& =-\frac{\sin k(L-x)}{k \sin k L} \int_{0}^{x} \sin k x^{\prime} f\left(x^{\prime}\right) d x^{\prime}-\frac{\sin k x}{k \sin k L} \int_{x}^{L} \sin k\left(L-x^{\prime}\right) f\left(x^{\prime}\right) d x^{\prime} .
\end{aligned}
$$

We can use the same Green's function to solve a different type of non-homogeneous string problem. Suppose that there is no bowing force, $f(x) \equiv 0$, but one end of the string is vibrated with frequency $\omega$ and amplitude $A$. This means that we will have boundary conditions $u(0)=0$ and $u(L)=A$. Equation (12.1.5) still applies as does the Green's function (12.1.14). In fact, inserting the latter along with the new boundary conditions on $u(x),(12.1 .5)$ gives us the solution $\psi(x, t)=u(x) e^{-i \omega t}$ with

$$
u(x)=\left.D \frac{d}{d x^{\prime}} G\left(x ; x^{\prime}\right)\right|_{x^{\prime}=L}=A \frac{1}{k \sin k L} \sin k x k \cos k(L-L)=A \frac{\sin k x}{\sin k L} .
$$


Notice that the Wronskian of $u_{<}(x)$ and $u_{>}(x)$ provides this particular Green's function with simple poles located at the zeros of $\sin k L$ which means at the values $k=\frac{\omega}{c}=$ $\frac{m \pi}{L}, m=1,2, \ldots$. As we know and as the last sub-section suggested should happen, these values correspond to the normal modes of vibration of the string. Thus, when we stimulate the string at values of $\omega$ approaching one of its natural frequencies, the Green's function and hence the transverse displacement of the string increases without limit. In other words, we produce a resonant response or resonance.

\subsubsection{Eigenfunction Expansions : The Bilinear Formula}

The origin of resonant behaviour and even of the Green's function method itself becomes much more transparent when one attempts solution by means of an eigenfunction expansion.

We shall use the modification to the non-homogeneous Sturm-Liouville equation that was introduced at the end of Section 12.1.2. Specifically, we shall write it in the form

$$
\mathfrak{L} u(x)+\lambda \rho(x) u(x)=f(x) \text { where } \mathfrak{L} \equiv \frac{d}{d x}\left(p(x) \frac{d}{d x}\right)-r(x)
$$

so that the correspondence with (12.1.1) is brought about by the replacement of $q(x)$ in that equation by $r(x)-\lambda \rho(x)$. Here $\lambda$ is a constant and $\rho(x)$ is the (positive-definite) weight function that is defined by the eigenvalue equation

$$
\mathfrak{L} u_{m}(x)=-\lambda_{m} \rho(x) u_{m}(x)
$$

and the (homogeneous) boundary conditions

$$
\alpha_{1} u_{m}(a)+\left.\alpha_{2} \frac{d u_{m}}{d x}\right|_{x=a}=0 \text { and } \beta_{1} u_{m}(b)+\left.\beta_{2} \frac{d u_{m}}{d x}\right|_{x=b}=0 \text {. }
$$

We know that the eigenfunctions $\left\{u_{m}(x)\right\}$ form a complete orthogonal set for the space of functions that are square integrable with respect to the weight function $\rho(x)$ over the interval $a \leq x \leq b$. Therefore, assuming the eigenfunctions to be normalized to 1 over $a \leq x \leq b$, we expand $u(x)$ and $\frac{f(x)}{\rho(x)}$ in terms of them and write

$$
u(x)=\sum_{m=1}^{\infty} a_{m} u_{m}(x) \text { where } a_{m}=\int_{a}^{b} u_{m}^{\star}\left(x^{\prime}\right) u\left(x^{\prime}\right) \rho\left(x^{\prime}\right) d x^{\prime},
$$

and

$$
f(x)=\rho(x) \sum_{m=1}^{\infty} b_{m} u_{m}(x) \text { where } b_{m}=\int_{a}^{b} u_{m}^{\star}\left(x^{\prime}\right) f\left(x^{\prime}\right) d x^{\prime}
$$


Notice that we are allowing for the possibility that the eigenfunctions are complex. Substituting these expansions into the DE in (12.1.15), we obtain the algebraic equation

$$
\rho(x) \sum_{m=1}^{\infty} a_{m}\left(\lambda-\lambda_{m}\right) u_{m}(x)=\rho(x) \sum_{m=1}^{\infty} b_{m} u_{m}(x) .
$$

Because of the orthogonality of the $\left\{u_{m}(x)\right\}$, this implies

$$
a_{m}=\frac{b_{m}}{\lambda-\lambda_{m}} \text { for each } m=1,2,3, \ldots
$$

Substituting back into the series in (12.1.18) and using the definition of $b_{m}$ in (12.1.19), this yields a solution to the non-homogeneous DE, namely

$$
u(x)=\sum_{m=1}^{\infty} \frac{u_{m}(x)}{\lambda-\lambda_{m}} \int_{a}^{b} u_{m}^{\star}\left(x^{\prime}\right) f\left(x^{\prime}\right) d x^{\prime}
$$

Both the integral and the series should be uniformly convergent and so we interchange their order to obtain

$$
u(x)=\int_{a}^{b} G\left(x ; x^{\prime}\right) f\left(x^{\prime}\right) d x^{\prime} \text { where } G\left(x ; x^{\prime}\right)=\sum_{m=1}^{\infty} \frac{u_{m}(x) u_{m}^{\star}\left(x^{\prime}\right)}{\lambda-\lambda_{m}} .
$$

This is called the bilinear formula for the Green's function.

Note that if $f\left(x^{\prime}\right)=\delta\left(x^{\prime}-x_{0}\right),(12.1 .20)$ yields the solution $u(x)=G\left(x ; x_{0}\right)$. This verifies that $G\left(x ; x^{\prime}\right)$ as defined by the bilinear formula is a solution of the Green's function DE

$$
\mathfrak{L} G\left(x ; x^{\prime}\right)+\lambda \rho(x) G\left(x ; x^{\prime}\right)=\delta\left(x-x^{\prime}\right) .
$$

Moreover, since each eigenfunction $u_{m}(x)$ satisfies the homogeneous boundary conditions (12.1.17), so does $G\left(x ; x^{\prime}\right)$.

We remarked at the beginning of this sub-section that the origin of resonant behaviour becomes particularly transparent when the bilinear formula is used to construct a Green's function. Indeed, it corresponds to $\lambda=\lambda_{m}$ for some $m$ and when that happens $G\left(x ; x^{\prime}\right)$ clearly becomes undefined and there is no solution to the original non-homogeneous problem unless, by chance,

$$
\int_{a}^{b} u_{m}^{\star}\left(x^{\prime}\right) f\left(x^{\prime}\right) d x^{\prime}=0
$$

for that particular value of $m$.

The normalized eigenfunctions of the bowed string differential operator, $\mathfrak{L} \equiv \frac{d^{2}}{d x^{2}}$, corresponding to the homogeneous boundary conditions $u(0)=u(L)=0$, are

$$
u_{m}(x)=\sqrt{\frac{2}{L}} \sin \frac{m \pi x}{L} \text { with } \lambda_{m}=\frac{m^{2} \pi^{2}}{L^{2}} \quad m=1,2, \ldots
$$


Therefore, substituting into (12.1.20) and using $\lambda=k^{2}$, we find the bilinear form

$$
G\left(x ; x^{\prime}\right)=\frac{2}{L} \sum_{m=1}^{\infty} \frac{\sin \frac{m \pi x}{L} \sin \frac{m \pi x^{\prime}}{L}}{k^{2}-\frac{m^{2} \pi^{2}}{L^{2}}}
$$

which exhibits explicitly the poles at $k=\frac{m \pi}{L}$. Evidently, this series is the Fourier sine series expansion of (12.1.14)

$$
G\left(x ; x^{\prime}\right)= \begin{cases}-\frac{\sin k x \sin k\left(L-x^{\prime}\right)}{k \sin k L} & \text { for } 0 \leq x<x^{\prime} \\ -\frac{\sin k x^{\prime} \sin k(L-x)}{k \sin k L} & \text { for } x^{\prime}<x \leq L\end{cases}
$$

\subsubsection{Application: the Infinite Stretched String}

In many problems that are amenable to use of the bilinear formula, the eigenvalue spectrum is continuous. To illustrate what happens in such a situation, we shall consider a one-dimensional analogue of acoustic and electromagnetic radiation.

Suppose that we have an infinitely long stretched string that is subjected to a transverse harmonic force per unit length $F(x, t)=-T f(x) e^{-i \omega_{0} t}$ where $T$ is, as usual, the tension in the string. Here, "infinitely long" means long enough that the ends of the string have a negligible effect on the behaviour of points in any neighbourhood of the middle. The PDE satisfied by the transverse displacement is the same as for the finite string,

$$
\frac{\partial^{2} \psi}{\partial x^{2}}-\frac{1}{c^{2}} \frac{\partial^{2} \psi}{\partial t^{2}}=-\frac{1}{T} F(x, t),
$$

but the boundary conditions are, of course, quite different. We shall assume that the displacement is everywhere bounded: $|\psi(x, t)|<\infty$ for all $-\infty<x<\infty$.

As with the finite string, we shall seek solutions of the form $\psi(x, t)=u(x) e^{-i \omega_{0} t}$ which reduces the problem to one of solving the non-homogeneous ODE

$$
\frac{d^{2} u}{d x^{2}}+k_{0}^{2} u(x)=f(x) \text { where } k_{0}=\frac{\omega_{0}}{c}
$$

subject to $|u(x)|<\infty$ for all $-\infty<x<\infty$. Therefore, the Green's function for this problem must be the solution of

$$
\frac{d^{2} G}{d x^{2}}+k_{0}^{2} G\left(x ; x^{\prime}\right)=\delta\left(x-x^{\prime}\right)
$$

that satisfies the same (homogeneous) boundary conditions. To find it by means of the bilinear formula, we must first solve the eigenvalue problem

$$
\frac{d^{2}}{d x^{2}} u_{\lambda}(x)=-\lambda u_{\lambda}(x) \text { with }\left|u_{\lambda}(x)\right|<\infty \text { for all }-\infty<x<\infty .
$$


The boundedness condition can only be met if $\lambda<0$. Therefore, we set $\lambda=-k^{2}$ and obtain the (normalized) eigenfunctions $u_{k}(x)=\frac{1}{\sqrt{2 \pi}} e^{-i k x}$. This means that the bilinear formula for our Green's function is

$$
G\left(x ; x^{\prime}\right)=\frac{1}{2 \pi} \int_{-\infty}^{\infty} \frac{e^{i k x^{\prime}} e^{-i k x}}{k_{0}^{2}-k^{2}} d k .
$$

Evidently, this is an inverse Fourier transform and so must be the solution of (12.1.22) that we would have obtained had we used Fourier transforms. To confirm this, we set $\mathcal{F}\left\{G\left(x ; x^{\prime}\right)\right\} \equiv g\left(k ; x^{\prime}\right)$, and transform (12.1.22) to obtain

$$
-k^{2} g\left(k ; x^{\prime}\right)+k_{0}^{2} g\left(k ; x^{\prime}\right)=\frac{1}{\sqrt{2 \pi}} \int_{-\infty}^{\infty} e^{i k x} \delta\left(x-x^{\prime}\right) d x=\frac{1}{\sqrt{2 \pi}} e^{i k x^{\prime}},
$$

or

$$
g\left(k ; x^{\prime}\right)=\frac{1}{\sqrt{2 \pi}} \frac{e^{i k x^{\prime}}}{k_{0}^{2}-k^{2}} .
$$

Thus, since

$$
G\left(x ; x^{\prime}\right)=\frac{1}{\sqrt{2 \pi}} \int_{-\infty}^{\infty} e^{-i k x} g\left(k ; x^{\prime}\right) d k,
$$

we do indeed recover the bilinear formula (12.1.24).

We have some experience in evaluating Fourier integrals and so (12.1.24) should lead to a closed form expression for $G\left(x ; x^{\prime}\right)$ that can be compared to the expression obtainable from the direct construction technique. There is a slight complication however: the integrand has (simple) poles on the real axis at $k= \pm k_{0}$. This means that we require an additional piece of information that instructs us how to deform the contour of integration to avoid them. What that information may be becomes apparent as soon as we investigate the residues at the poles. The residues at $k= \pm k_{0}$ are

$$
\mp \frac{1}{4 \pi} \frac{e^{ \pm i k\left(x^{\prime}-x\right)}}{k_{0}}
$$

respectively. The first of these would make a contribution to $G\left(x ; x^{\prime}\right) e^{-i \omega_{0} t}$ that contains the factor $e^{-i\left[k_{0}\left(x-x^{\prime}\right)+\omega_{0} t\right]}$ while the contribution from the second would contain $e^{i\left[k_{0}\left(x-x^{\prime}\right)-\omega_{0} t\right]}$. These are waves travelling to the left and to the right, respectively, from the source point $x=x^{\prime}$. But the role of the Green's function is to give us the the response at a point $x$ due to a disturbance at a point $x^{\prime}$. Therefore, if $x$ is to the left of $x^{\prime}, x<x^{\prime}$, then $G\left(x ; x^{\prime}\right)$ should not include the wave travelling to the right. Consequently, the contour should not enclose the pole $k=-k_{0}$. On the other hand, if $x$ is to the right of $x^{\prime}, x>x^{\prime}$, the wave travelling to the left must be excluded and so now the contour should not enclose $k=k_{0}$. Thus, the physical identity of the Green's function provides a key piece of information. We shall now complement it with the requirements of Jordan's Lemma to come up with a unique prescription for the contour 
of integration. If $x<x^{\prime}$, we are obliged to close the contour in the upper half plane and so we avoid the poles by means of small semi-circle closing above $k=-k_{0}$ and a second semi-circle closing below $k=k_{0}$. If $x>x^{\prime}$, the contour is closed in the lower half-plane and so the poles are avoided by exactly the same means.

The evaluation of the Fourier integral is now straightforward: one finds

$$
G\left(x ; x^{\prime}\right)=2 \pi i\left[-\frac{1}{2 \pi} \frac{e^{i k\left(x^{\prime}-x\right)}}{k+k_{0}}\right]_{k=k_{0}}=-\frac{i}{2 k_{0}} e^{i k_{0}\left(x^{\prime}-x\right)} \text { for } x<x^{\prime},
$$

and

$$
G\left(x ; x^{\prime}\right)=2 \pi i\left[\frac{1}{2 \pi} \frac{e^{i k\left(x^{\prime}-x\right)}}{k-k_{0}}\right]_{k=-k_{0}}=-\frac{i}{2 k_{0}} e^{-i k_{0}\left(x^{\prime}-x\right)} \text { for } x>x^{\prime},
$$

or

$$
G\left(x ; x^{\prime}\right)=-\frac{i}{2 k_{0}} e^{i k_{0}\left|x-x^{\prime}\right|} \text { for all }-\infty<x<\infty .
$$

Therefore, the solution to the infinite string problem is $\psi(x, t)=u(x) e^{-i \omega_{0} t}$ where

$$
u(x)=\int_{-\infty}^{\infty} G\left(x ; x^{\prime}\right) f\left(x^{\prime}\right) d x^{\prime}=-\frac{i}{2 k_{0}}\left\{\int_{-\infty}^{x} e^{i k_{0}\left(x-x^{\prime}\right)} f\left(x^{\prime}\right) d x^{\prime}+\int_{x}^{\infty} e^{i k_{0}\left(x^{\prime}-x\right)} f\left(x^{\prime}\right) d x^{\prime}\right\}
$$

Notice that if we had chosen the time dependence to be $e^{i \omega_{0} t}$, we would have had the reverse correspondence between the waves travelling to the left and right. In that case, the Green's function to be used is the complex conjugate of the one in (12.1.25)

If the applied force is real, $F(x, t)=-T f(x)\left\{\begin{array}{c}\cos \omega_{0} t \\ \sin \omega_{0} t\end{array}\right\}, f^{\star}(x)=f(x)$, we can express the transverse displacement in an explicitly real form by setting it equal to the real or imaginary parts, respectively of $u(x) e^{-i \omega_{0} t}$ (the real part yielding an even function of $t$ and the imaginary part an odd function of $t$ to match the parity of $\cos \omega_{0} t$ and $\left.\sin \omega_{0} t\right)$. In the first case, this yields

$$
\psi(x, t)=\int_{-\infty}^{\infty} \operatorname{Re}\left\{e^{-i \omega_{0} t} G\left(x ; x^{\prime}\right)\right\} f\left(x^{\prime}\right) d x^{\prime}=\frac{1}{2 k_{0}} \int_{-\infty}^{\infty} \sin \left(k_{0}\left|x-x^{\prime}\right|-\omega_{0} t\right) f\left(x^{\prime}\right) d x^{\prime}
$$

and in the second,

$$
\psi(x, t)=\int_{-\infty}^{\infty} \operatorname{Im}\left\{e^{-i \omega_{0} t} G\left(x ; x^{\prime}\right)\right\} f\left(x^{\prime}\right) d x^{\prime}=-\frac{1}{2 k_{0}} \int_{-\infty}^{\infty} \cos \left(\left|x-x^{\prime}\right|-\omega_{0} t\right) f\left(x^{\prime}\right) d x^{\prime} .
$$


We shall complete our analysis of the infinite stretched string by constructing the Green's function directly. The relevant homogeneous DE is

$$
\frac{d^{2} u}{d x^{2}}+k_{0}^{2} u(x)=0
$$

We require a solution $u_{<}(x)$ which meets the boundary condition that as $x \rightarrow-\infty$ $u_{<}(x) e^{-i \omega_{0} t}$ is a wave travelling to the left. Since the general solution is $\left\{\begin{array}{c}e^{i k_{0} x} \\ e^{-i k_{0} x}\end{array}\right\}$, this means that $u_{<}(x)=e^{-i k_{0} x}$. Next, we seek a solution $u_{>}(x)$ such that as $x \rightarrow+\infty$ $u_{>}(x) e^{-i \omega_{0} t}$ is a wave travelling to the right. The obvious choice is $u_{>}(x)=e^{i k_{0} x}$.

The Wronskian of $u_{<}(x)$ and $u_{>}(x)$ is

$$
W(x)=u_{<}(x) \frac{d u_{>}}{d x}-\frac{d u_{>}}{d x} u_{<}(x)=i 2 k_{0} .
$$

Thus, since $p(x) \equiv 1$, equation (12.1.12) yields

$$
G\left(x ; x^{\prime}\right)=-\frac{i}{2 k_{0}} \begin{cases}e^{i k_{0}\left(x-x^{\prime}\right)} & \text { for }-\infty<x<x^{\prime} \\ e^{i k_{0}\left(x^{\prime}-x\right)} & \text { for } x^{\prime}<x<\infty\end{cases}
$$

or,

$$
G\left(x ; x^{\prime}\right)=-\frac{i}{2 k_{0}} e^{i k_{0}\left|x-x^{\prime}\right|} \text { for all }-\infty<x<\infty .
$$

in full agreement with (12.1.25).

\subsection{Partial Differential Equations}

\subsubsection{Green's Theorem and Its Consequences}

In more than one dimension a non-homogeneous boundary value problem generally involves the solution of a PDE

$$
\mathfrak{L} \psi(\boldsymbol{r})=f(\boldsymbol{r}) \text { with } \mathfrak{L} \equiv \nabla \cdot[p(\boldsymbol{r}) \nabla]+s(\boldsymbol{r})
$$

inside a volume $V$ that is bounded by a surface $S$ on which either $\psi(\boldsymbol{r})$ or $\boldsymbol{n} \cdot \boldsymbol{\nabla} \psi$ is specified. The partial differential operator $\mathfrak{L}$ in (12.2.1) is self-adjoint and satisfies Green's Theorem which states that if $u(\boldsymbol{r})$ and $v(\boldsymbol{r})$ are any two twice-differentiable functions,

$$
\int_{V}[u(\boldsymbol{r}) \mathfrak{L} v(\boldsymbol{r})-v(\boldsymbol{r}) \mathfrak{L} u(\boldsymbol{r})] d V=\int_{S} p(\boldsymbol{r})[u(\boldsymbol{r}) \nabla v(\boldsymbol{r})-v(\boldsymbol{r}) \nabla u(\boldsymbol{r})] \cdot d \boldsymbol{S} .
$$

The proof of the theorem follows from a consideration of the integrals 


$$
\begin{aligned}
\int_{S}[u(\boldsymbol{r}) p(\boldsymbol{r}) \nabla v(\boldsymbol{r})] \cdot d \boldsymbol{S} & =\int_{V} \nabla \cdot[u(\boldsymbol{r}) p(\boldsymbol{r}) \nabla v(\boldsymbol{r})] d V \\
& =\int_{V}(\nabla u(\boldsymbol{r})) \cdot(p(\boldsymbol{r}) \nabla v(\boldsymbol{r})) d V+\int_{V} u(\boldsymbol{r}) \nabla \cdot(p(\boldsymbol{r}) \nabla) v(\boldsymbol{r}) d V
\end{aligned}
$$

and

$$
\begin{aligned}
\int_{S}[v(\boldsymbol{r}) p(\boldsymbol{r}) \nabla u(\boldsymbol{r})] \cdot d \boldsymbol{S} & =\int_{V} \nabla \cdot[v(\boldsymbol{r}) p(\boldsymbol{r}) \nabla u(\boldsymbol{r})] d V \\
& =\int_{V}(\nabla v(\boldsymbol{r})) \cdot(p(\boldsymbol{r}) \nabla u(\boldsymbol{r})) d V+\int_{V} v(\boldsymbol{r}) \nabla \cdot(p(r) \nabla) u(\boldsymbol{r}) d V
\end{aligned}
$$

where we have used the divergence theorem in the first line of both equations. Subtracting the second from the first of these equations, there is a cancellation that gives us

$$
\int_{S} p(\boldsymbol{r})[u(\boldsymbol{r}) \nabla v(\boldsymbol{r})-v(\boldsymbol{r}) \nabla u(\boldsymbol{r})] \cdot d \boldsymbol{S}=\int_{V}[u(\boldsymbol{r}) \nabla \cdot(p(\boldsymbol{r}) \nabla) v(\boldsymbol{r})-v(\boldsymbol{r}) \nabla \cdot(p(\boldsymbol{r}) \nabla) u(\boldsymbol{r})] d V .
$$

Adding $u(\boldsymbol{r}) s(\boldsymbol{r}) v(\boldsymbol{r})-v(\boldsymbol{r}) s(\boldsymbol{r}) u(\boldsymbol{r})$ to the integrand on the left hand side completes the derivation of (12.2.2).

Let us introduce a Green's function $G\left(\boldsymbol{r} ; \boldsymbol{r}^{\prime}\right)$ by defining it to be a solution of

$$
\mathfrak{L} G\left(\boldsymbol{r} ; \boldsymbol{r}^{\prime}\right)=\delta\left(\boldsymbol{r}-\boldsymbol{r}^{\prime}\right)
$$

in $V$ subject to suitable boundary conditions on $S$. Applying Green's Theorem with $u(\boldsymbol{r})$ replaced by $\psi(\boldsymbol{r})$ (the solution of (12.2.1)) and $v(\boldsymbol{r})$ by $G\left(\boldsymbol{r} ; \boldsymbol{r}^{\prime}\right)$ and using their respective PDE's, we find

$$
\begin{aligned}
\int_{V}\left[\psi(\boldsymbol{r}) \mathfrak{L} G\left(\boldsymbol{r} ; \boldsymbol{r}^{\prime}\right)-G\left(\boldsymbol{r} ; \boldsymbol{r}^{\prime}\right) \mathfrak{L} \psi(\boldsymbol{r})\right] d V=\int_{V}\left[\psi(\boldsymbol{r}) \delta\left(\boldsymbol{r}-\boldsymbol{r}^{\prime}\right)-G\left(\boldsymbol{r} ; \boldsymbol{r}^{\prime}\right) f(\boldsymbol{r})\right] d V \\
=\psi\left(\boldsymbol{r}^{\prime}\right)-\int_{V} G\left(\boldsymbol{r} ; \boldsymbol{r}^{\prime}\right) f(\boldsymbol{r}) d V=\int_{S} p(\boldsymbol{r})\left[\psi(\boldsymbol{r}) \nabla G\left(\boldsymbol{r} ; \boldsymbol{r}^{\prime}\right)-G\left(\boldsymbol{r} ; \boldsymbol{r}^{\prime}\right) \nabla \psi(\boldsymbol{r})\right] \cdot d \boldsymbol{S} .
\end{aligned}
$$

Interchanging $\boldsymbol{r}$ and $\boldsymbol{r}^{\prime}$ and rearranging terms, the last two lines of this equation become

$$
\psi(\boldsymbol{r})=\int_{V} G\left(\boldsymbol{r}^{\prime} ; \boldsymbol{r}\right) f\left(\boldsymbol{r}^{\prime}\right) d V^{\prime}+\int_{S} p\left(\boldsymbol{r}^{\prime}\right)\left[\psi\left(\boldsymbol{r}^{\prime}\right) \nabla^{\prime} G\left(\boldsymbol{r}^{\prime} ; \boldsymbol{r}\right)-G\left(\boldsymbol{r}^{\prime} ; \boldsymbol{r}\right) \nabla^{\prime} \psi\left(\boldsymbol{r}^{\prime}\right)\right] \cdot d \boldsymbol{S}^{\prime}
$$


which is the multi-dimensional analogue of equation (12.1.5).

We now choose boundary conditions for $G\left(\boldsymbol{r} ; \boldsymbol{r}^{\prime}\right)$ that will eliminate unknown quantities from the surface integral on the right hand side of (10.2.4). Normally, there are only two cases to consider.

\section{Case 1 (Dirichlet Boundary Conditions): $\psi(r)$ is given on $S$.}

The obvious choice for the Green's function under this circumstance is the homogeneous condition

$$
G\left(\boldsymbol{r} ; \boldsymbol{r}^{\prime}\right)=0 \text { for } \boldsymbol{r} \text { on } S \text {. }
$$

Equation (12.2.4) then becomes

$$
\psi(\boldsymbol{r})=\int_{V} G\left(\boldsymbol{r}^{\prime} ; \boldsymbol{r}\right) f\left(\boldsymbol{r}^{\prime}\right) d V^{\prime}+\int_{S} p\left(\boldsymbol{r}^{\prime}\right) \psi\left(\boldsymbol{r}^{\prime}\right) \nabla^{\prime} G\left(\boldsymbol{r}^{\prime} ; \boldsymbol{r}\right) \cdot d \boldsymbol{S}^{\prime} .
$$

\section{Case 2 (Neumann Boundary Conditions): $n \cdot \nabla \psi(r)$ is given on $S$.}

The choice here is not quite so obvious. If we apply the divergence theorem to

$$
\int_{V} \mathfrak{L} G\left(\boldsymbol{r} ; \boldsymbol{r}^{\prime}\right) d V=\int_{V} \delta\left(\boldsymbol{r}-\boldsymbol{r}^{\prime}\right) d V=1,
$$

we find

$$
\int_{S} p(\boldsymbol{r}) \nabla G\left(\boldsymbol{r} ; \boldsymbol{r}^{\prime}\right) \cdot d \boldsymbol{S}+\int_{V} s(\boldsymbol{r}) G\left(\boldsymbol{r} ; \boldsymbol{r}^{\prime}\right) d V=1 .
$$

This means that if $s(\boldsymbol{r}) \equiv 0$, we cannot require $\boldsymbol{n} \cdot \boldsymbol{\nabla} G\left(\boldsymbol{r} ; \boldsymbol{r}^{\prime}\right)=0$ for $\boldsymbol{r}$ on $S$ since that would produce a contradiction. Therefore, we are obliged to recognize two sub-cases: if $s(\boldsymbol{r}) \neq 0$, we make the obvious choice and impose the homogeneous condition

$$
\boldsymbol{n} \cdot \nabla G\left(\boldsymbol{r} ; \boldsymbol{r}^{\prime}\right)=0 \text { for } \boldsymbol{r} \text { on } S
$$

and if $s(\boldsymbol{r}) \equiv 0$, we require the next best thing,

$$
\boldsymbol{n} \cdot \nabla G\left(\boldsymbol{r} ; \boldsymbol{r}^{\prime}\right)=\frac{1}{A_{p}}, \text { where } A_{p}=\int_{S} p(\boldsymbol{r}) d S, \text { for } \boldsymbol{r} \text { on } S .
$$

In the first instance, (12.2.4) becomes

$$
\psi(\boldsymbol{r})=\int_{V} G\left(\boldsymbol{r}^{\prime} ; \boldsymbol{r}\right) f\left(\boldsymbol{r}^{\prime}\right) d V^{\prime}-\int_{S} p\left(\boldsymbol{r}^{\prime}\right) G\left(\boldsymbol{r}^{\prime} ; \boldsymbol{r}\right) \nabla^{\prime} \psi\left(\boldsymbol{r}^{\prime}\right) \cdot d \boldsymbol{S}^{\prime}
$$


and in the second,

$$
\psi(\boldsymbol{r})=\langle\psi\rangle_{S}+\int_{V} G\left(\boldsymbol{r}^{\prime} ; \boldsymbol{r}\right) f\left(\boldsymbol{r}^{\prime}\right) d V^{\prime}-\int_{S} G\left(\boldsymbol{r}^{\prime} ; r\right) \nabla^{\prime} \psi\left(r^{\prime}\right) \cdot d \boldsymbol{S}^{\prime}
$$

where $\langle\psi\rangle_{S}=\frac{1}{A_{p}} \int_{S} p\left(\boldsymbol{r}^{\prime}\right) \psi\left(\boldsymbol{r}^{\prime}\right) d S^{\prime}$ is the weighted average of $\psi(\boldsymbol{r})$ over the whole surface $\boldsymbol{S}$.

Poisson's equation is an important example of a PDE for which $s(\boldsymbol{r}) \equiv 0$ and whose Green's function must therefore meet the non-homogeneous Neumann boundary condition (12.2.8). In that case, $\langle\psi\rangle_{S}=\frac{1}{A} \int_{S} \psi\left(\boldsymbol{r}^{\prime}\right) d S^{\prime}$ where $A$ is the area of the surface $S$. This means that $\psi(\boldsymbol{r})$ is determined only to within an additive constant by the boundary condition. On the other hand, we know from electromagnetic theory that the definition of zero potential is arbitrary and exercising that arbitrariness, we can set $\langle\psi\rangle_{S}$ to zero.

Now that we know how to solve for $\psi(\boldsymbol{r})$ in terms of $G\left(\boldsymbol{r} ; \boldsymbol{r}^{\prime}\right)$, it is time to turn our attention to the construction of Green's functions in more than one dimension. We will do so by considering specific PDE's and boundary conditions. But first, we shall deduce a property common to all Green's functions. What is involved is another application of Green's Theorem. Setting $u(\boldsymbol{r})=G\left(\boldsymbol{r} ; \boldsymbol{r}^{\prime}\right)$ and $v(\boldsymbol{r})=G\left(\boldsymbol{r} ; \boldsymbol{r}^{\prime \prime}\right)$ in equation (12.2.2), we note that the surface term vanishes and leaves us with the result $G\left(\boldsymbol{r}^{\prime} ; \boldsymbol{r}^{\prime \prime}\right)$ $G\left(\boldsymbol{r}^{\prime \prime} ; \boldsymbol{r}^{\prime}\right)=0$ or,

$$
G\left(\boldsymbol{r}^{\prime} ; \boldsymbol{r}^{\prime \prime}\right)=G\left(\boldsymbol{r}^{\prime \prime} ; \boldsymbol{r}^{\prime}\right) .
$$

In other words, $G\left(\boldsymbol{r} ; \boldsymbol{r}^{\prime}\right)$ is symmetric under $\boldsymbol{r} \leftrightarrow \boldsymbol{r}^{\prime}$.

\subsubsection{Poisson's Equation in Two Dimensions and With Rectangular Symmetry}

Suppose that we wish to find the static deflection $u(x, y)$ of a rectangular membrane due to an external force. Using $f(x, y)$ to denote the external force per unit area divided by the tension, this will require that we solve

$$
\frac{\partial^{2} u}{\partial x^{2}}+\frac{\partial^{2} u}{\partial y^{2}}=f(x, y)
$$

subject to (homogeneous) Dirichlet conditions at the fixed edges which we shall locate at $x=0, x=a, y=0$ and $y=b$.

The Green's function for this problem is the solution of

$$
\frac{\partial^{2} G}{\partial x^{2}}+\frac{\partial^{2} G}{\partial y^{2}}=\delta\left(x-x^{\prime}\right) \delta\left(y-y^{\prime}\right)
$$

that satisfies

$$
G\left(0, y ; x^{\prime}, y^{\prime}\right)=G\left(a, y ; x^{\prime}, y^{\prime}\right)=G\left(x, 0 ; x^{\prime}, y^{\prime}\right)=G\left(x, b ; x^{\prime}, y^{\prime}\right)=0 .
$$


In general, it is either not possible or not useful to find a closed form expression for a multi-dimensional Green's function when the boundaries are finite closed surfaces. However, as in the one-dimensional case, there are two construction methods: the eigenfunction expansion and the direct construction approach. The first of these yields an expression, the bilinear formula, with as many summations or integrations as there are dimensions (or separable differential operators in $\mathfrak{L}$ ). The second eliminates one of these summations by making use of our ability to directly construct closed form expressions for one dimensional Green's functions.

\section{Eigenfunction Expansion Method:}

Let $u_{\lambda}(x, y)$ denote the normalized eigenfunctions of $\nabla^{2}$ that satisfy homogeneous Dirichlet conditions at $x=0, x=a, y=0$ and $y=b$.

In other words, let

$$
\begin{array}{r}
\nabla^{2} u_{\lambda}(x, y)=-\lambda u_{\lambda}(x, y) \text { with }\left\|u_{\lambda}(x, y)\right\|=1 \\
\text { and } u_{\lambda}(0, y)=u_{\lambda}(a, y)=u_{\lambda}(x, 0)=u_{\lambda}(x, b)=0 .
\end{array}
$$

Since their closure relation must be

$$
\sum_{\lambda} u_{\lambda}(x, y) u_{\lambda}^{\star}\left(x^{\prime}, y^{\prime}\right)=\delta\left(x-x^{\prime}\right) \delta\left(y-y^{\prime}\right)
$$

it is clear that the bilinear formula of Section 12.1.3 applies here too and yields

$$
G\left(x, y ; x^{\prime}, y^{\prime}\right)=\sum_{\lambda} \frac{u_{\lambda}(x, y) u_{\lambda}^{*}\left(x^{\prime}, y^{\prime}\right)}{-\lambda} .
$$

We know already (from Section 10.8) that the eigenvalues for the rectangular membrane are $\lambda_{m, n}=\frac{m^{2} \pi^{2}}{a^{2}}+\frac{n^{2} \pi^{2}}{b^{2}}, m, n=1,2,3, \ldots$ corresponding to the (normalized) eigenfunctions $u_{m, n}(x, y)=\frac{2}{\sqrt{a b}} \sin \frac{m \pi x}{a} \sin \frac{n \pi y}{b}$. Therefore, the bilinear formula representation of our green's function is

$$
G\left(x, y ; x^{\prime}, y^{\prime}\right)=-\frac{4}{a b} \sum_{m=1}^{\infty} \sum_{n=1}^{\infty} \frac{\sin \frac{m \pi x}{a} \sin \frac{n \pi y}{b} \sin \frac{m \pi x^{\prime}}{a} \sin \frac{n \pi y^{\prime}}{b}}{\frac{m^{2} \pi^{2}}{a^{2}}+\frac{n^{2} \pi^{2}}{b^{2}}} .
$$

\section{Direct Construction Method:}

We start with a partial eigenfunction expansion. Choosing to do so in the $y$ variable, we write

$$
G\left(x, y ; x^{\prime}, y^{\prime}\right)=\sqrt{\frac{2}{b}} \sum_{n=1}^{\infty} G_{n}\left(x ; x^{\prime}, y^{\prime}\right) \sin \frac{n \pi y}{b}
$$


and invoke closure for the normalized eigenfunctions $\sqrt{\frac{2}{b}} \sin \frac{n \pi y}{b}$,

$$
\delta\left(y-y^{\prime}\right)=\frac{2}{b} \sum_{n=1}^{\infty} \sin \frac{n \pi y}{b} \sin \frac{n \pi y^{\prime}}{b} .
$$

Substituting these two expansions into the Green's function PDE and equating on a term by term basis, we conclude that $G_{n}\left(x ; x^{\prime}, y^{\prime}\right)$ factors according to

$$
G_{n}\left(x ; x^{\prime}, y^{\prime}\right)=\sqrt{\frac{2}{b}} \sin \frac{n \pi y^{\prime}}{b} g_{n}\left(x ; x^{\prime}\right) \text { where } \frac{d^{2} g_{n}}{d x^{2}}-\frac{n^{2} \pi^{2}}{b^{2}} g_{n}\left(x ; x^{\prime}\right)=\delta\left(x-x^{\prime}\right) .
$$

This is a one-dimensional Green's function DE which we can solve by the direct construction method.

Remembering that we have homogeneous boundary conditions, $g_{n}\left(0 ; x^{\prime}\right)=$ $g_{n}\left(a ; x^{\prime}\right)=0$, we proceed by seeking a solution $u_{<}(x)$ of the homogeneous DE

$$
\frac{d^{2} u}{d x^{2}}-\frac{n^{2} \pi^{2}}{b^{2}} u(x)=0
$$

that satisfies $u_{<}(0)=0$. Since the general solution is $u(x)=\left\{\begin{array}{c}\cosh \frac{n \pi x}{b} \\ \sinh \frac{n \pi x}{b}\end{array}\right\}$, the simplest choice is $u_{<}(x)=\sinh \frac{n \pi x}{b}$.

Next, we need a solution $u_{>}(x)$ of the homogeneous DE that satisfies $u_{>}(a)=0$. Again, the simplest choice is pretty obvious: $u_{>}(x)=\sinh \frac{n \pi}{b}(x-a)$.

The Wronskian of $u_{<}(x)$ and $u_{>}(x)$ is

$$
W(x)=u_{<}(x) u_{>}^{\prime}(x)-u_{>}(x) u_{<}^{\prime}(x)=\frac{n \pi}{b} \sinh \frac{n \pi a}{b} .
$$

Therefore, using (12.1.12) we have

$$
g_{n}\left(x ; x^{\prime}\right)= \begin{cases}-\frac{b}{n \pi} \frac{\sinh \frac{n \pi x}{b} \sinh \frac{n \pi}{b}\left(a-x^{\prime}\right)}{\sinh \frac{n \pi a}{b}}, & 0 \leq x<x^{\prime} \\ -\frac{b}{n \pi} \frac{\sinh \frac{n \pi}{b}(a-x) \sinh \frac{n \pi x^{\prime}}{b}}{\sinh \frac{n \pi a}{b}}, & x^{\prime}<x \leq a\end{cases}
$$

Substituting back into the expansion (12.2.19) for $G\left(x, y ; x^{\prime}, y^{\prime}\right)$ we conclude that

$$
G\left(x, y ; x^{\prime}, y^{\prime}\right)=\left\{\begin{array}{l}
-\sum_{n=1}^{\infty} \frac{2}{n \pi} \frac{\sinh \frac{n \pi x}{b} \sinh \frac{n \pi}{b}\left(a-x^{\prime}\right)}{\sinh \frac{n \pi a}{b}} \sin \frac{n \pi y}{b} \sin \frac{n \pi y^{\prime}}{b} \\
-\sum_{n=1}^{\infty} \frac{2}{n \pi} \frac{\sinh \frac{n \pi}{b}(a-x) \sinh \frac{n \pi x^{\prime}}{b}}{\sinh \frac{n \pi a}{b}} \sin \frac{n \pi y}{b} \sin \frac{n \pi y^{\prime}}{b}
\end{array}\right.
$$

for $\left\{\begin{array}{l}0 \leq x<x^{\prime} \\ x^{\prime}<x \leq a\end{array}\right.$, respectively. This is equivalent to the double Fourier representation (12.2.18) found by application of the bilinear formula but with the sum over $m$ actually performed. 
Had our starting point been substitution of

$$
\delta\left(x-x^{\prime}\right)=\frac{2}{a} \sum_{m=1}^{\infty} \sin \frac{m \pi x}{a} \sin \frac{m \pi x^{\prime}}{a},
$$

and

$$
G\left(x, y ; x^{\prime}, y^{\prime}\right)=\sqrt{\frac{2}{a}} \sum_{m=1}^{\infty} G_{m}\left(y ; x^{\prime}, y^{\prime}\right) \sin \frac{m \pi x}{a}
$$

into the PDE (12.2.13), we would have found $G_{m}\left(y ; x^{\prime}, y^{\prime}\right)=g_{m}\left(y ; y^{\prime}\right) \sin \frac{m \pi x^{\prime}}{a}$ where

$$
g_{m}\left(y ; y^{\prime}\right)= \begin{cases}-\frac{a}{m \pi} \frac{\sinh \frac{m \pi y}{a} \sinh \frac{m \pi}{a}\left(b-y^{\prime}\right)}{\sinh \frac{m \pi b}{a}}, & 0 \leq y<y^{\prime} \\ -\frac{a}{m \pi} \frac{\sinh \frac{m \pi}{a}(b-y) \sinh \frac{m \pi y^{\prime}}{a}}{\sinh \frac{m \pi b}{a}}, & y^{\prime}<y \leq b\end{cases}
$$

This, of course, yields an expression for $G\left(x, y ; x^{\prime}, y^{\prime}\right)$ that is equivalent to summing over $n$ in the double Fourier series (12.2.18). It could have been obtained directly from (12.2.22) by invoking the problem's symmetry under $x \leftrightarrow y,\left(x^{\prime} \leftrightarrow y^{\prime}\right)$, and $a \leftrightarrow b$.

\subsubsection{Potential Problems in Three Dimensions and the Method of Images}

Coulomb's Law is an implicit expression of the solution of the Green's function PDE

$$
\nabla^{2} G\left(\boldsymbol{r} ; \boldsymbol{r}^{\prime}\right)=\delta\left(\boldsymbol{r}-\boldsymbol{r}^{\prime}\right)
$$

plus the (Dirichlet) boundary condition $\lim _{r \rightarrow \infty} G\left(\boldsymbol{r} ; \boldsymbol{r}^{\prime}\right)=0$. It tells us that the potential due to unit charge located at the point $r \begin{array}{r}r \rightarrow \infty \\ =\boldsymbol{r}^{\prime}\end{array}$ is

$$
\psi(\boldsymbol{r})=-\frac{1}{4 \pi \varepsilon_{0}} \frac{1}{\left|\boldsymbol{r}-\boldsymbol{r}^{\prime}\right|}
$$

and, since the charge density associated with the charge is $\rho(\boldsymbol{r})=\delta\left(\boldsymbol{r}-\boldsymbol{r}^{\prime}\right)$, this means that

$$
\nabla^{2} \psi(\boldsymbol{r})=-\frac{\rho(\boldsymbol{r})}{\varepsilon_{0}}=-\frac{1}{\varepsilon_{0}} \delta\left(\boldsymbol{r}-\boldsymbol{r}^{\prime}\right) .
$$

Comparing this with (12.2.24), we deduce that

$$
G\left(\boldsymbol{r} ; \boldsymbol{r}^{\prime}\right)=-\frac{1}{4 \pi} \frac{1}{\left|\boldsymbol{r}-\boldsymbol{r}^{\prime}\right|}
$$

This is confirmed by direct integration of (12.2.24). Integrating over a spherical volume centred at $\boldsymbol{r}=\boldsymbol{r}^{\prime}$ and applying the divergence theorem, we find

$$
\int_{S} \nabla G\left(\boldsymbol{r} ; \boldsymbol{r}^{\prime}\right) \cdot d \mathbf{S}=1
$$


where $S$ denotes the boundary surface of the sphere. But the normal gradient on a spherical surface is just the partial derivative with respect to the radial coordinate. Therefore, we can rewrite this last equation as

$$
\int_{S} \frac{\partial G}{\partial r} d S=\int_{0}^{2 \pi} \int_{0}^{\pi} \frac{\partial G}{\partial r} r^{2} \sin \theta d \theta d \varphi=1
$$

where $r=\left|\boldsymbol{r}-\boldsymbol{r}^{\prime}\right|$. With the origin of coordinates at $\boldsymbol{r}=\boldsymbol{r}^{\prime}$, the delta function in the PDE and the boundary condition to be imposed on its solution both depend only on the radial variable and so the same must be true of the solution itself. Thus, the integration can be performed to give us

$$
\frac{d G}{d r}=\frac{1}{4 \pi r^{2}}=\frac{1}{4 \pi} \frac{1}{\left|\boldsymbol{r}-\boldsymbol{r}^{\prime}\right|^{2}}
$$

Integrating once more and using the boundary condition to dispose of the integration constant, we obtain, as expected,

$$
G\left(\boldsymbol{r} ; \boldsymbol{r}^{\prime}\right)=-\frac{1}{4 \pi} \frac{1}{\left|\boldsymbol{r}-\boldsymbol{r}^{\prime}\right|}
$$

This result can also be obtained by use of Fourier transforms as will be demonstrated in Section 12.3.3.

If the Dirichlet condition is imposed on a finite surface, we can still solve for the Green's function in closed form by using a trick that is called, in electrostatic theory, the method of images. Any Green's function can be set equal to a superposition of solutions of the non-homogeneous and homogeneous PDE's. In the present case, this means that we can set

$$
G\left(\boldsymbol{r} ; \boldsymbol{r}^{\prime}\right)=G_{S}\left(\boldsymbol{r} ; \boldsymbol{r}^{\prime}\right)+G_{o}\left(\boldsymbol{r} ; \boldsymbol{r}^{\prime}\right) \text { where } G_{s}\left(\boldsymbol{r} ; \boldsymbol{r}^{\prime}\right)=-\frac{1}{4 \pi} \frac{1}{\left|\boldsymbol{r}-\boldsymbol{r}^{\prime}\right|}
$$

and

$$
\nabla^{2} G_{o}\left(\boldsymbol{r} ; \boldsymbol{r}^{\prime}\right)=0 \text { with }\left.\left[G_{s}\left(\boldsymbol{r} ; \boldsymbol{r}^{\prime}\right)+G_{o}\left(\boldsymbol{r} ; \boldsymbol{r}^{\prime}\right)\right]\right|_{\boldsymbol{r} \text { on surface }}=0 .
$$

For specificity, let us take the boundary surface to be a sphere of radius $R$. If $\boldsymbol{r}$ is constrained to vary inside the spherical volume, $r<R$, and $\boldsymbol{r}^{\prime \prime}$ is a point outside, $r^{\prime \prime}>R$, the delta function $\delta\left(\boldsymbol{r}-\boldsymbol{r}^{\prime \prime}\right)$ is zero and $\frac{1}{\left|\boldsymbol{r}-\boldsymbol{r}^{\prime \prime}\right|}$ is a solution of the homogeneous PDE there. This means we can set

$$
G_{o}\left(\boldsymbol{r} ; \boldsymbol{r}^{\prime}\right)=-\frac{1}{4 \pi} \frac{k}{\left|\boldsymbol{r}-\boldsymbol{r}^{\prime \prime}\right|}, \quad r \leq R \text { and } r^{\prime \prime}>R,
$$

where $k$ is a constant and $\boldsymbol{r}^{\prime \prime}$ is chosen to lie along the same radius vector as $\boldsymbol{r}^{\prime}$. The values of $k$ and $r^{\prime \prime}$ are to be determined by imposing the boundary condition (12.2.27).

Since $\boldsymbol{r}^{\prime \prime}=\frac{r^{\prime \prime}}{r^{\prime}} \boldsymbol{r}^{\prime}$, our Green's function is

$$
G\left(\boldsymbol{r} ; \boldsymbol{r}^{\prime}\right)=G_{s}\left(\boldsymbol{r} ; \boldsymbol{r}^{\prime}\right)+G_{0}\left(\boldsymbol{r} ; \boldsymbol{r}^{\prime}\right)=-\frac{1}{4 \pi}\left[\frac{1}{\left|\boldsymbol{r}-\boldsymbol{r}^{\prime}\right|}+\frac{k}{\left|\boldsymbol{r}-\frac{r^{\prime \prime}}{r^{\prime}} \boldsymbol{r}^{\prime}\right|}\right]
$$


This vanishes at $r=R$ if

$$
r^{\prime \prime}=\frac{R^{2}}{r^{\prime}} \text { and } k=-\frac{r^{\prime \prime}}{R}
$$

which gives us the unique solution

$$
G\left(\boldsymbol{r} ; \boldsymbol{r}^{\prime}\right)=-\frac{1}{4 \pi}\left[\frac{1}{\left|\boldsymbol{r}-\boldsymbol{r}^{\prime}\right|}+\frac{R}{r^{\prime}} \frac{1}{\left|\boldsymbol{r}-\frac{r R^{2}}{r^{\prime 2}} \boldsymbol{r}^{\prime}\right|}\right], r \text { and } r^{\prime} \leq R .
$$

Visual inspection of this result reveals why its construction is called the method of images. We know that $G_{s}\left(\boldsymbol{r} ; \boldsymbol{r}^{\prime}\right)$ has the physical significance of the potential due to an isolated point charge located at $\boldsymbol{r}=\boldsymbol{r}^{\prime}$. Similarly, the Green's function we are trying to find is the potential due to that same charge when it is enclosed within a conducting sphere of radius $R$. What we have found is that the effect of the sphere is the same as that of adding an "image" point charge located at the inverse of $\boldsymbol{r}=\boldsymbol{r}^{\prime}$ with respect to the spherical surface.

This approach works well for any simple boundary surface, a plane and a cylinder of infinite length being two other examples. However, finding image points is a challenge with more complicated surfaces and is generally not worth the effort. Even (12.2.28) is difficult to work with in the context of finding the potential due to a continuous charge distribution via (12.2.6). A more fruitful approach is to proceed as we did in Section 12.2.2 and expand the Green's function in series (or integrals).

\subsubsection{Expansion of the Dirichlet Green's Function for Poisson's Equation When There Is Spherical Symmetry}

Suppose that we have Dirichlet conditions imposed on a surface consisting of two concentric spheres of radii $r=a$ and $r=b, b>a$. This means that we wish to solve

$$
\nabla^{2} G\left(\boldsymbol{r} ; \boldsymbol{r}^{\prime}\right)=\delta\left(\boldsymbol{r}-\boldsymbol{r}^{\prime}\right) \text { subject to }\left.G\left(\boldsymbol{r} ; \boldsymbol{r}^{\prime}\right)\right|_{r=a}=\left.G\left(\boldsymbol{r} ; \boldsymbol{r}^{\prime}\right)\right|_{r=b}=0 .
$$

In spherical coordinates, the delta function in the PDE can be expanded according to

$$
\begin{aligned}
\delta\left(\boldsymbol{r}-\boldsymbol{r}^{\prime}\right) & =\frac{1}{r^{2}} \delta\left(r-r^{\prime}\right) \delta\left(\cos \theta-\cos \theta^{\prime}\right) \delta\left(\varphi-\varphi^{\prime}\right) \\
& =\frac{1}{r^{2}} \delta\left(r-r^{\prime}\right) \sum_{l=0}^{\infty} \sum_{m=-l}^{l}\left(Y_{l}^{m}\left(\theta^{\prime}, \varphi\right)\right)^{\star} Y_{l}^{m}(\theta, \varphi)
\end{aligned}
$$

where we have used the closure relation for spherical harmonics. Similarly, we can write

$$
G\left(\boldsymbol{r} ; \boldsymbol{r}^{\prime}\right)=\sum_{l=0}^{\infty} \sum_{m=-l}^{l} G_{l m}\left(r ; \boldsymbol{r}^{\prime}\right) Y_{l}^{m}(\theta, \varphi)
$$


and substitute both expansions into the PDE of (12.2.29) to obtain

$$
\begin{gathered}
\sum_{l=0}^{\infty} \sum_{m=-l}^{l}\left\{\frac{1}{r} \frac{d^{2}}{d r^{2}}\left(r G_{l m}\left(r ; \boldsymbol{r}^{\prime}\right)-\frac{l(l+1)}{r^{2}} G_{l m}\left(r ; \boldsymbol{r}^{\prime}\right)\right\} Y_{l}^{m}(\theta, \varphi)\right. \\
=\frac{1}{r^{2}} \delta\left(r-r^{\prime}\right) \sum_{l=0}^{\infty} \sum_{m=-l}^{l}\left(Y_{l}^{m}\left(\theta^{\prime}, \varphi^{\prime}\right)\right)^{\star} Y_{l}^{m}(\theta, \varphi) .
\end{gathered}
$$

Invoking the orthogonality of the spherical harmonics to set up equations on a term by term basis, we deduce that $G_{l m}\left(r ; \boldsymbol{r}^{\prime}\right)$ must factor according to

$$
G_{l m}\left(r ; \boldsymbol{r}^{\prime}\right)=g_{l}\left(r ; r^{\prime}\right) Y_{l}^{m}\left(\theta^{\prime}, \varphi^{\prime}\right)
$$

where

$$
r^{2} \frac{d^{2}}{d r^{2}} g_{l}\left(r ; r^{\prime}\right)+2 r \frac{d}{d r} g_{l}\left(r ; r^{\prime}\right)-l(l+1) g_{l}\left(r ; r^{\prime}\right)=\delta\left(\boldsymbol{r}-\boldsymbol{r}^{\prime}\right) .
$$

The homogeneous counterpart of (12.2.34) is Euler's equation

$$
r^{2} \frac{d^{2} u}{d r^{2}}+2 r \frac{d u}{d r}-l(l+1) u(r)=0
$$

which has the general solution $\left\{\begin{array}{c}r^{l} \\ r^{-l-1}\end{array}\right\}$. Therefore, a solution $u_{<}(r)$ that satisfies the boundary condition $u_{<}(a)=0$ is

$$
u<(r)=\left(r^{l}-\frac{a^{2 l+1}}{r^{l+1}}\right), \quad a \leq r
$$

while one that satisfies $u_{>}(b)=0$ is

$$
u>(r)=\left(\frac{1}{r^{l+1}}-\frac{r^{l}}{b^{2 l+1}}\right), \quad r \leq b .
$$

The Wronskian of $u_{<}(r)$ and $u_{>}(r)$ is

$$
W(r)=u_{<}(r) u_{>}^{\prime}(r)-u_{>}(r) u_{<}^{\prime}(r)=\frac{2 l+1}{r^{2}}\left[\left(\frac{a}{b}\right)^{2 l+1}-1\right]
$$

and the Sturm-Liouville function $p(r)$ is $r^{2}$. Thus, from the direct construction formula (12.1.12) for one-dimensional Green's functions, we have

$$
g_{l}\left(r ; r^{\prime}\right)=\frac{(-1)}{(2 l+1)\left[1-\left(\frac{a}{b}\right)^{2 l+1}\right]} \begin{cases}\left(r^{l}-\frac{a^{2 l+1}}{r^{l+1}}\right)\left(\frac{1}{r^{\prime l+1}}-\frac{r^{\prime l}}{b^{2 l+1}}\right), & a \leq r<r^{\prime} \\ \left(\frac{1}{r^{l+1}}-\frac{r^{l}}{b^{2 l+1}}\right)\left(r^{\prime l}-\frac{a^{2 l+1}}{r^{\prime l+1}}\right), & r^{\prime}<r \leq b\end{cases}
$$

or,

$$
g_{l}\left(r ; r^{\prime}\right)=\frac{(-1)}{(2 l+1)\left[1-\left(\frac{a}{b}\right)^{2 l+1}\right]}\left(r_{>}^{l}-\frac{a^{2 l+1}}{r_{<}^{l+1}}\right)\left(\frac{1}{r_{>}^{l+1}}-\frac{r_{>}^{l}}{b^{2 l+1}}\right)
$$


where $r_{<} \equiv$ the smaller of $r$ and $r^{\prime}$ and $r>\equiv$ the larger of $r$ and $r^{\prime}$. Substituting this into (12.2.33) and the latter into (12.2.31) gives us a final expression for the Poisson equation Green's function for a spherical shell bounded by $r=a$ and $r=b$ :

$$
G\left(\boldsymbol{r} ; \boldsymbol{r}^{\prime}\right)=-\sum_{l=0}^{\infty} \sum_{m=-l}^{l} \frac{Y_{l}^{m}(\theta, \varphi)\left(Y_{l}^{m}\left(\theta^{\prime}, \varphi^{\prime}\right)\right)^{\star}}{(2 l+1)\left[1-\left(\frac{a}{b}\right)^{2 l+1}\right]}\left(r_{<}^{l}-\frac{a^{2 l+1}}{r_{<}^{l+1}}\right)\left(\frac{1}{r_{>}^{l+1}}\right) .
$$

There are three special cases:

1. if $a=0$ and $b \rightarrow \infty$, we have

$$
G\left(\boldsymbol{r} ; \boldsymbol{r}^{\prime}\right)=\sum_{l=0}^{\infty} \sum_{m=-l}^{l} \frac{(-1)}{2 l+1} \frac{r_{<}^{l}}{r_{>}^{l+1}} Y_{l}^{m}(\theta, \varphi)\left(Y_{l}^{m}\left(\theta^{\prime}, \varphi^{\prime}\right)\right)^{\star}=-\frac{1}{4 \pi} \frac{1}{\left|\boldsymbol{r}-\boldsymbol{r}^{\prime}\right|}
$$

where the last equality was derived earlier as an application of the addition theorem of spherical harmonics;

2. if $a$ remains finite and $b \rightarrow \infty$, we have an exterior problem and the Green's function is

$$
G\left(\boldsymbol{r} ; \boldsymbol{r}^{\prime}\right)=\sum_{l=0}^{\infty} \sum_{m=-l}^{l} \frac{(-1)}{2 l+1} \frac{1}{r_{>}^{2 l+1}}\left(r_{<}^{l}-\frac{a^{2 l+1}}{r_{<}^{l+1}}\right) Y_{l}^{m}(\theta, \varphi)\left(Y_{l}^{m}\left(\theta^{\prime}, \varphi^{\prime}\right)\right)^{\star} ;
$$

3. if $a=0$ and $b$ remains finite, we have an interior problem and the Green's function is

$$
G\left(\boldsymbol{r} ; \boldsymbol{r}^{\prime}\right)=\sum_{l=0}^{\infty} \sum_{m=-l}^{l} \frac{(-1)}{2 l+1} r_{<}^{l}\left(\frac{1}{r>^{l+1}}\right) Y_{l}^{m}(\theta, \varphi)\left(Y_{l}^{m}\left(\theta^{\prime}, \varphi^{\prime}\right)\right)^{\star} .
$$

\subsubsection{Applications}

\section{Solution of Laplace's Equation}

We know already that the potential inside a sphere of radius $b$ with no charges present but subject to $\psi(b, \theta, \varphi)=V(\theta, \varphi)$ is

$$
\psi(r, \theta, \varphi)=\sum_{l=0}^{\infty} \sum_{m=-l}^{l} c_{l m} r^{l} Y_{l}^{m}(\theta, \varphi)
$$

with

$$
c_{l m}=\frac{1}{b^{l}} \int_{0}^{2 \pi} \int_{-1}^{1}\left(Y_{l}^{m}\left(\theta^{\prime}, \varphi^{\prime}\right)^{\star} V\left(\theta^{\prime}, \varphi^{\prime}\right) d\left(\cos \theta^{\prime}\right) d \varphi^{\prime} .\right.
$$

What we wish to verify now is that the Green's function we have just derived yields exactly the same solution. To do so, we require the normal gradient

$$
\left.\boldsymbol{n} \cdot \nabla G\left(\boldsymbol{r} ; \boldsymbol{r}^{\prime}\right)\right|_{r=b}=\left.\frac{\partial}{\partial r} G\left(\boldsymbol{r} ; \boldsymbol{r}^{\prime}\right)\right|_{r=b} \text {. }
$$


From (12.2.39) we have

$$
\left.\frac{\partial}{\partial r} G\left(\boldsymbol{r} ; \boldsymbol{r}^{\prime}\right)\right|_{r=b}=\left.\frac{\partial}{\partial r_{>}} G\left(\boldsymbol{r} ; \boldsymbol{r}^{\prime}\right)\right|_{r_{>}=b}=\frac{1}{b^{2}} \sum_{l=0}^{\infty} \sum_{m=-l}^{l}\left(\frac{r}{b}\right)^{l}\left(Y_{l}^{m}\left(\theta^{\prime}, \varphi^{\prime}\right)\right)^{*} Y_{l}^{m}(\theta, \varphi) .
$$

Thus, since $\rho(\boldsymbol{r}) \equiv 0$ and $d S^{\prime}=b^{2} d\left(\cos \theta^{\prime}\right) d \varphi^{\prime}$, we obtain from equation (12.2.9)

$$
\begin{aligned}
\psi(\boldsymbol{r}) & =-\frac{1}{\varepsilon_{0}} \int_{V} G\left(\boldsymbol{r} ; \boldsymbol{r}^{\prime}\right) \rho\left(\boldsymbol{r}^{\prime}\right) d V^{\prime}+\int_{S} \psi\left(\boldsymbol{r}^{\prime}\right) \boldsymbol{n} \cdot \nabla^{\prime} G\left(\boldsymbol{r} ; \boldsymbol{r}^{\prime}\right) d S^{\prime} \\
& =\sum_{l=0}^{\infty} \sum_{m=-l}^{l}\left[\int_{0}^{2 \pi} \int_{-1}^{1} V\left(\theta^{\prime}, \varphi^{\prime}\right)\left(Y_{l}^{m}\left(\theta^{\prime}, \varphi^{\prime}\right)\right)^{\star} d\left(\cos \theta^{\prime}\right) d \varphi^{\prime}\right]\left(\frac{r}{b}\right)^{l} Y_{l}^{m}(\theta, \varphi)
\end{aligned}
$$

which is identical to our earlier result as required.

Note that if $V(\theta, \varphi)$ is independent of $\varphi$ (that is, if we have azimuthal symmetry), only the $m=0$ terms are retained in $G\left(\boldsymbol{r} ; \boldsymbol{r}^{\prime}\right)$. Thus, since

$$
Y_{l}^{m}(\theta, \varphi)\left(Y_{l}^{m}\left(\theta^{\prime}, \varphi^{\prime}\right)\right)^{\star} \rightarrow \frac{2 l+1}{4 \pi} P_{l}(\cos \theta) P_{l}\left(\cos \theta^{\prime}\right) \text { for } m=0,
$$

the electrostatic potential becomes

$$
\psi(r, \theta)=\sum_{l=0}^{\infty}\left[\frac{2 l+1}{2} \int_{-1}^{1} V\left(\theta^{\prime}\right) P_{l}\left(\cos \theta^{\prime}\right) d\left(\cos \theta^{\prime}\right)\right]\left(\frac{r}{b}\right)^{l} P_{l}(\cos \theta) .
$$

\section{Solution of Poisson's Equation}

Consider a hollow, grounded sphere of radius $b$ with a concentric ring of charge, of radius $c<b$ and total charge $Q$, inside it. Taking the ring to lie in the $x y$-plane, we can assert that the charge density inside the sphere will be independent of $\varphi$ and have a delta function dependence on $r$ and $\theta$. Thus, we can write

$$
\rho(\boldsymbol{r})=A \delta(r-c) \delta(\cos \theta) \text { where } \mathrm{A} \text { is a constant of proportionality. }
$$

The constant $A$ can be determined from the normalizing condition

$$
Q=\int_{0}^{b} \int_{0}^{\pi} \int_{0}^{2 \pi} \rho(\boldsymbol{r}) r^{2} d r \sin \theta d \theta d \varphi
$$

Thus, $A=\frac{Q}{2 \pi c^{2}}$. This means that the electrostatic potential inside the sphere is the solution of

$$
\nabla^{2} \psi(\boldsymbol{r})=-\frac{Q}{2 \pi c^{2} \varepsilon_{0}} \delta(r-c) \delta(\cos \theta)
$$

that satisfies $\psi(b, \theta, \varphi)=0$. 
Using the interior problem Green's function (12.2.39) modified for azimuthal symmetry, we have

$$
\psi(\boldsymbol{r})=\int_{0}^{2 \pi} \int_{-1}^{1} \int_{0}^{b}\left[-\frac{Q}{2 \pi c^{2} \varepsilon_{0}} \delta\left(r^{\prime}-c\right) \delta\left(\cos \theta^{\prime}\right)\right] G\left(\boldsymbol{r} ; \boldsymbol{r}^{\prime}\right) r^{\prime 2} d r^{\prime} d\left(\cos \theta^{\prime}\right) d \varphi^{\prime}
$$

and so,

$$
\psi(\boldsymbol{r})=\sum_{l=0}^{\infty} \frac{P_{l}(\cos \theta)}{4 \pi} \int_{0}^{2 \pi} \int_{-1}^{1} \int_{0}^{b} \frac{Q}{2 \pi c^{2} \varepsilon_{0}} \delta\left(r^{\prime}-c\right) \delta\left(\cos \theta^{\prime}\right) \times r_{<}^{l}\left(\frac{1}{r_{>}^{l+1}}\right) r^{\prime 2} d r^{\prime} d\left(\cos \theta^{\prime}\right) d \varphi^{\prime}
$$

or,

$$
\psi(\boldsymbol{r})=\frac{Q}{4 \pi \varepsilon_{0}} \sum_{l=0}^{\infty} P_{l}(0) r_{<}^{l}\left(\frac{1}{r_{>}^{l+1}}\right) P_{l}(\cos \theta)
$$

where $r_{<}\left(r_{>}\right)$is now the smaller (larger) of $r$ and $c$. Making use of

$$
P_{2 l+1}(0)=0 \text { and } P_{2 l}(0)=\frac{(-1)^{l}(2 l) !}{2^{2 l}(l !)^{2}},
$$

this becomes

$$
\psi(\boldsymbol{r})=\frac{Q}{4 \pi \varepsilon_{0}} \sum_{l=0}^{\infty} \frac{(-1)^{l}(2 l) !}{2^{2 l}(l !)^{2}} r_{<}^{2 l}\left(\frac{1}{r_{>}^{2 l+1}}\right) P_{2 l}(\cos \theta) .
$$

Notice that for $b \rightarrow \infty$ and $r>c$, this reduces to

$$
\psi(\boldsymbol{r})=\frac{Q}{4 \pi \varepsilon_{0}} \sum_{l=0}^{\infty} \frac{(-1)^{l}(2 l) !}{2^{2 l}(l !)^{2}} \frac{c^{2 l}}{r^{2 l+1}} P_{2 l}(\cos \theta)
$$

which for a point on the $\mathrm{z}$-axis converges to the well-known consequence of Coulomb's law,

$$
\psi(z, 0)=\frac{Q}{4 \pi \varepsilon_{0}} \frac{1}{\sqrt{z^{2}+c^{2}}} .
$$

\subsection{The Non-Homogeneous Wave and Diffusion Equations}

\subsubsection{The Non-Homogeneous Helmholtz Equation}

The non-homogeneous wave equation is

$$
\nabla^{2} \psi(\boldsymbol{r}, t)-\frac{1}{c^{2}} \frac{\partial^{2} \psi(\boldsymbol{r}, t)}{\partial t^{2}}=\sigma(\boldsymbol{r}, t)
$$

As in the bowed string problem, if the source is monochromatic and harmonic, that is if $\sigma(\boldsymbol{r}, t)=\sigma(\boldsymbol{r}) e^{-i \omega t}$, we can assume the same time dependence for $\psi(\boldsymbol{r}, t)$, 
$\psi(\boldsymbol{r}, t)=\psi(\boldsymbol{r}) e^{-i \omega t}$. This means that the problem is effectively time-independent and so no initial conditions are needed. We proceed by substitution into the original PDE which yields

$$
\left(\nabla^{2}+k^{2}\right) \psi(\boldsymbol{r})=\sigma(\boldsymbol{r}), \quad k=\omega / c .
$$

From equation (12.2.4) we know that the solution of the non-homogeneous Helmholtz equation is

$$
\psi(\boldsymbol{r})=\int_{V} G\left(\boldsymbol{r}^{\prime} ; \boldsymbol{r}\right) \sigma\left(\boldsymbol{r}^{\prime}\right) d V^{\prime}+\int_{S}\left[\psi\left(\boldsymbol{r}^{\prime}\right) \boldsymbol{n} \cdot \nabla^{\prime} G\left(\boldsymbol{r}^{\prime} ; \boldsymbol{r}\right)-G\left(\boldsymbol{r}^{\prime} ; \boldsymbol{r}\right) \boldsymbol{n} \cdot \nabla^{\prime} \psi\left(\boldsymbol{r}^{\prime}\right)\right] d S^{\prime}
$$

where

$$
\left(\nabla^{2}+k^{2}\right) G\left(\boldsymbol{r} ; \boldsymbol{r}^{\prime}\right)=\delta\left(\boldsymbol{r}-\boldsymbol{r}^{\prime}\right)
$$

and $G\left(\boldsymbol{r} ; \boldsymbol{r}^{\prime}\right)$ is subject to the homogeneous counterparts of whatever boundary conditions are imposed on $\psi(\boldsymbol{r})$. One expression for $G\left(\boldsymbol{r} ; \boldsymbol{r}^{\prime}\right)$ is provided by the bilinear formula,

$$
G\left(\boldsymbol{r} ; \boldsymbol{r}^{\prime}\right)=\sum_{n} \frac{u_{n}(\boldsymbol{r})\left(u_{n}\left(\boldsymbol{r}^{\prime}\right)\right)^{*}}{k^{2}-k_{n}^{2}}
$$

where the functions $u_{n}(\boldsymbol{r})$ are the normalized normal modes defined by $\left(\nabla^{2}+k^{2}\right) u_{n}(\boldsymbol{r})=0$ with boundary conditions $u_{n}(\boldsymbol{r})=0$ or $\boldsymbol{n} \cdot \nabla u_{n}(\boldsymbol{r})=0$ for $\boldsymbol{r}$ on $S$. Another is provided by the same kind of partial expansion and direct construction technique that we used for Poisson's equation.

\subsubsection{The Forced Drumhead}

If the external force per unit area applied normal to a drumhead is $F(\boldsymbol{r}, t)$, its transverse displacement will obey the equation

$$
\nabla^{2} \psi(\boldsymbol{r}, t)-\frac{1}{c^{2}} \frac{\partial^{2}}{\partial t^{2}} \psi(\boldsymbol{r}, t)=-\frac{1}{c^{2} \mu} F(\boldsymbol{r}, t) \equiv f(\boldsymbol{r}, t)
$$

where, as before, $\mu$ is the mass per unit area. We assume that $f(\boldsymbol{r}, t)=f(\boldsymbol{r}) e^{-i \omega t}$ and set the forced displacement of the drumhead equal to $\psi(\boldsymbol{r}, t)=\psi(\boldsymbol{r}) e^{-i \omega t}$. Substituting into (10.3.5), we get the two dimensional non-homogeneous Helmholtz equation

$$
\left(\nabla^{2}+k^{2}\right) \psi(\boldsymbol{r})=f(\boldsymbol{r}), \quad k=\frac{\omega}{c} .
$$

This is to be solved subject to the (Dirichlet) boundary condition $\psi(\boldsymbol{r})=0$ for $\boldsymbol{r}$ on the perimeter of the drumhead. Thus, if the drumhead is circular with radius $a$, we have the condition $\psi(a, \theta)=0$. 
With both $\psi(\boldsymbol{r})$ and $G\left(\boldsymbol{r} ; \boldsymbol{r}^{\prime}\right)$ satisfying homogeneous Dirichlet conditions, the solution is provided by the integral

$$
\psi(\boldsymbol{r})=\int_{0}^{2 \pi} \int_{0}^{a} G\left(\boldsymbol{r} ; \boldsymbol{r}^{\prime}\right) f\left(\boldsymbol{r}^{\prime}\right) r^{\prime} d r^{\prime} d \theta^{\prime}
$$

Thus, all we need to do is construct the Green's function.

Trying the direct construction method first, we expand $G\left(\boldsymbol{r} ; \boldsymbol{r}^{\prime}\right)$ in a Fourier series in $\theta$ :

$$
G\left(\boldsymbol{r} ; \boldsymbol{r}^{\prime}\right)=\sum_{m=-\infty}^{\infty} G_{m}\left(\boldsymbol{r} ; \boldsymbol{r}^{\prime}, \theta^{\prime}\right) e^{i m \theta}
$$

Next, we use closure to expand the delta function in $\theta$ :

$$
\delta\left(\boldsymbol{r}-\boldsymbol{r}^{\prime}\right)=\frac{1}{r} \delta\left(r-r^{\prime}\right) \delta\left(\theta-\theta^{\prime}\right)=\frac{1}{r} \delta\left(r-r^{\prime}\right) \frac{1}{2 \pi} \sum_{m=-\infty}^{\infty} e^{i m\left(\theta-\theta^{\prime}\right)} .
$$

Substituting these expansions into (12.3.4) expressed in terms of two dimensional polar coordinates and using the orthogonality of the Fourier functions, we find that the coefficients $G_{m}\left(\boldsymbol{r} ; \boldsymbol{r}^{\prime}, \theta^{\prime}\right)$ factor according to

$$
G_{m}\left(\boldsymbol{r} ; r^{\prime}, \theta^{\prime}\right)=\frac{1}{2 \pi} e^{-i m \theta^{\prime}} g_{m}\left(r ; r^{\prime}\right)
$$

where

$$
\frac{d^{2}}{d r^{2}} g_{m}\left(r ; r^{\prime}\right)+\frac{1}{r} \frac{d}{d r} g_{m}\left(r ; r^{\prime}\right)+\left(k^{2}-\frac{m^{2}}{r^{2}}\right) g_{m}\left(r ; r^{\prime}\right)=\frac{1}{r} \delta\left(r-r^{\prime}\right) .
$$

The homogeneous version of this is Bessel's equation with general solution $u(r)=$ $\left\{\begin{array}{c}J_{|m|}(k r) \\ N_{|m|}(k r)\end{array}\right\}$. A solution that satisfies the boundary condition $\left|u_{<}(0)\right|<\infty$ is $u_{<}(r)=J_{|m|}(k r), 0 \leq r$, while one that satisfies $u_{>}(a)=0$ is $u_{>}(r)=N_{|m|}(k a) J_{|m|}(k r)-$ $J_{|m|}(k a) N_{|m|}(k r), r \leq a$. Their Wronskian is

$$
W(r)=-k J_{|m|}(k a)\left[J_{|m|}(k r) N_{|m|}^{\prime}(k r)-N_{|m|}(k r) J_{|m|}^{\prime}(k r)\right] .
$$

We can show from their small $x$ behaviour that the Wronskian of the Bessel and Neumann functions is

$$
J_{m}(x) N_{m}^{\prime}(x)-J_{m}^{\prime}(x) N_{m}(x)=\frac{2}{\pi x} .
$$

Therefore, $W(r)=-\frac{2}{\pi r} J_{|m|}(k a)$ which with $p(r)=r$ and equation (12.1.12) gives us

$$
g_{m}\left(r ; r^{\prime}\right)=-\frac{\pi}{2} \frac{1}{J_{|m|}(k a)} \begin{cases}J_{|m|}(k r)\left[N_{|m|}(k a) J_{|m|}\left(k r^{\prime}\right)-J_{|m|}(k a) N_{|m|}\left(k r^{\prime}\right)\right], & 0 \leq r<r^{\prime} \\ {\left[N_{|m|}(k a) J_{|m|}(k r)-J_{|m|}(k a) N_{|m|}(k r)\right] J_{|m|}\left(k r^{\prime}\right),} & r^{\prime}<r \leq a\end{cases}
$$


Substituting back into (12.3.7), this yields the Green's function

$$
\begin{aligned}
G\left(\boldsymbol{r} ; \boldsymbol{r}^{\prime}\right)= & \frac{1}{4} \frac{J_{0}\left(k r_{<}\right)}{J_{0}(k a)}\left[J_{0}(k a) N_{0}\left(k r_{>}\right)-N_{0}(k a) J_{0}\left(k r_{>}\right)\right] \\
& +\frac{1}{2} \sum_{m=1}^{\infty} \frac{J_{m}\left(k r_{<}\right)}{J_{m}(k a)}\left[J_{m}(k a) N_{m}\left(k r_{>}\right)-N_{m}(k a) J_{m}\left(k r_{>}\right)\right] \cos m\left(\theta-\theta^{\prime}\right)
\end{aligned}
$$

where, as usual, $r_{<}\left(r_{>}\right)$is the smaller (larger) of $r$ and $r^{\prime}$.

To find the equivalent bilinear formula expression for the Green's function is quite straight forward since we already know what are the circular drumhead eigenfunctions or normal modes. Specifically, from Section 11.3.2 we have

$$
u_{m n}(r, \theta)=\left\{\begin{array}{l}
J_{m}\left(k_{m n} r\right) \cos m \theta \\
J_{m}\left(k_{m n} r\right) \sin m \theta
\end{array} \quad m=0,1,2, \ldots, \quad n=1,2, \ldots\right.
$$

To normalize these functions over the area of the drumhead, we multiply them by the normalization constants for the Bessel and Fourier functions which means multiplying them by

$$
N_{0 n}=\frac{1}{\sqrt{\pi} a J_{0}^{\prime}\left(k_{0 n} a\right)} \text { for } m=0 \text { and } N_{m n}=\sqrt{\frac{2}{\pi}} \frac{1}{a J_{m}^{\prime}\left(k_{m n} a\right)} \text { for } m=1,2, \ldots
$$

Substituting into (12.3.5), we obtain the bilinear formula

$$
\begin{aligned}
G\left(\boldsymbol{r} ; \boldsymbol{r}^{\prime}\right)= & \frac{1}{\pi a^{2}} \sum_{n=1}^{\infty} \frac{1}{\left[J_{0}^{\prime}\left(k_{0 n} a\right)\right]^{2}} \frac{J_{0}\left(k_{0 n} r\right) J_{0 n}\left(k_{0 n} r^{\prime}\right)}{k^{2}-k_{0 n}^{2}} \\
& +\frac{2}{\pi a^{2}} \sum_{m=1}^{\infty} \sum_{n=1}^{\infty} \frac{1}{\left[J_{m}^{\prime}\left(k_{m n} a\right)\right]^{2}} \frac{J_{m}\left(k_{m n} r\right) J_{m}\left(k_{m n} r^{\prime}\right) \cos m\left(\theta-\theta^{\prime}\right)}{k^{2}-k_{m n}^{2}}
\end{aligned}
$$

where we have used the identity $\cos m \theta \cos m \theta^{\prime}+\sin m \theta \sin m \theta^{\prime}=\cos m\left(\theta-\theta^{\prime}\right)$.

\subsubsection{The Non-Homogeneous Helmholtz Equation With Boundaries at Infinity}

When there are no finite boundaries (the source is isolated), the boundary condition that accompanies the Green's function PDE

$$
\left(\nabla^{2}+k^{2}\right) G\left(\boldsymbol{r} ; \boldsymbol{r}^{\prime}\right)=\delta\left(\boldsymbol{r}-\boldsymbol{r}^{\prime}\right)
$$

is simply that the solution be bounded and, in particular, that $\lim _{\left|\boldsymbol{r}-\boldsymbol{r}^{\prime}\right| \rightarrow \infty}\left|G\left(\boldsymbol{r} ; \boldsymbol{r}^{\prime}\right)\right|<\infty$. This can be solved by using Fourier transforms in much the same way that we did in Section 12.1.5. 
We start by putting a subscript on the wave number in the Green's function PDE to distinguish it from the transform variable:

$$
\left(\nabla^{2}+k_{0}^{2}\right) G\left(\boldsymbol{r} ; \boldsymbol{r}^{\prime}\right)=\delta\left(\boldsymbol{r}-\boldsymbol{r}^{\prime}\right)
$$

and so the time dependence associated with $G\left(\boldsymbol{r} ; \boldsymbol{r}^{\prime}\right)$ is now $e^{-i \omega_{0} t}, \omega_{0}=c k_{0}$. Taking the three-dimensional transform of (12.3.13), we obtain

$$
g\left(\boldsymbol{k} ; \boldsymbol{r}^{\prime}\right)=\frac{1}{(2 \pi)^{3 / 2}} \frac{e^{i \boldsymbol{k} \cdot \boldsymbol{r}^{\prime}}}{k_{0}^{2}-k^{2}}
$$

where $\boldsymbol{k}^{2}=\boldsymbol{k} \cdot \boldsymbol{k}$ and

$$
g\left(\boldsymbol{k} ; \boldsymbol{r}^{\prime}\right) \equiv \mathcal{F}\left\{G\left(\boldsymbol{r} ; \boldsymbol{r}^{\prime}\right)\right\}=\frac{1}{(2 \pi)^{3 / 2}} \int_{0}^{2 \pi} \int_{0}^{\pi} \int_{0}^{\infty} e^{i \boldsymbol{k} \cdot \boldsymbol{r}} G\left(\boldsymbol{r} ; \boldsymbol{r}^{\prime}\right) r^{2} d r \sin \theta d \theta d \varphi .
$$

Thus,

$$
G\left(\boldsymbol{r} ; \boldsymbol{r}^{\prime}\right)=\frac{1}{(2 \pi)^{3}} \int_{0}^{2 \pi} \int_{0}^{\pi} \int_{0}^{\infty} \frac{e^{i \boldsymbol{k} \cdot\left(\boldsymbol{r}^{\prime}-\boldsymbol{r}\right)}}{k_{0}^{2}-k^{2}} k^{2} d k \sin \theta_{k} d \theta_{k} d \varphi_{k}
$$

which we recognize as the bilinear formula for the Green's function.

To evaluate this integral, we take the $k_{3}$ axis in the direction of $\boldsymbol{r}-\boldsymbol{r}^{\prime}$ so that

$$
\boldsymbol{k} \cdot\left(\boldsymbol{r}-\boldsymbol{r}^{\prime}\right)=k R \cos \theta_{k}, \quad R=\left|\boldsymbol{r}-\boldsymbol{r}^{\prime}\right|
$$

Then,

$$
\begin{aligned}
G\left(\boldsymbol{r} ; \boldsymbol{r}^{\prime}\right) & =-\frac{1}{(2 \pi)^{3}} \int_{0}^{2 \pi} \int_{0}^{\pi} \int_{0}^{\infty} \frac{e^{-i k R \cos \theta_{k}}}{k^{2}-k_{0}^{2}} k^{2} d k \sin \theta_{k} d \theta_{k} d \varphi_{k} \\
& =-\frac{1}{(2 \pi)^{2}} \frac{2}{R} \int_{0}^{\infty} \frac{\sin k R}{k^{2}-k_{0}^{2}} k d k \\
& =-\frac{1}{(2 \pi)^{2}} \frac{1}{i R} \int_{0}^{\infty} \frac{e^{i k R}-e^{-i k R}}{k^{2}-k_{0}^{2}} k d k \\
& =-\frac{1}{(2 \pi)^{2}} \frac{1}{i R} \int_{-\infty}^{\infty} \frac{e^{i k R}}{k^{2}-k_{0}^{2}} k d k .
\end{aligned}
$$

The final integral can be evaluated by contour integration. When combined with time dependence $e^{-i \omega_{0} t}$, inclusion of the pole at $k=-k_{0}$ within the contour results in an incoming wave. On the other hand, the residue of the pole at $k=k_{0}$ yields an outgoing 
wave. Therefore, we use the same contours that were used in Section 12.1.5 and obtain the solutions

$$
G_{\text {out }}\left(\boldsymbol{r} ; \boldsymbol{r}^{\prime}\right)=-\frac{1}{4 \pi} \frac{e^{i k_{0}\left|\boldsymbol{r}-\boldsymbol{r}^{\prime}\right|}}{\left|\boldsymbol{r}-\boldsymbol{r}^{\prime}\right|}
$$

and

$$
G_{i n}\left(\boldsymbol{r} ; \boldsymbol{r}^{\prime}\right)=-\frac{1}{4 \pi} \frac{e^{-i k_{0}\left|\boldsymbol{r}-\boldsymbol{r}^{\prime}\right|}}{\left|\boldsymbol{r}-\boldsymbol{r}^{\prime}\right|}
$$

These are used to generate purely outgoing or incoming wave solutions, respectively. Thus, for example, if the source $\sigma(\boldsymbol{r}, t)=\sigma(\boldsymbol{r}) e^{-i \omega_{0} t}$ is an isolated loudspeaker or acoustic antenna, the waves that it emits will be described by

$$
\psi(\boldsymbol{r}, t)=-\frac{e^{-i \omega_{0} t}}{4 \pi} \int_{\text {all }} \frac{e^{i k_{0}\left|\boldsymbol{r}-\boldsymbol{r}^{\prime}\right|}}{\left|\boldsymbol{r}-\boldsymbol{r}^{\prime}\right|} \sigma(\boldsymbol{r}) d V^{\prime} .
$$

Far from the source, $r \gg r^{\prime}$, we have $\left|\boldsymbol{r}-\boldsymbol{r}^{\prime}\right| \sim r-\boldsymbol{n} \cdot \boldsymbol{r}^{\prime}$ where $\boldsymbol{n}=\frac{\boldsymbol{r}}{r}$ and so $\frac{e^{i k_{0}\left|r-r^{\prime}\right|}}{\left|\boldsymbol{r}-\boldsymbol{r}^{\prime}\right|} \sim \frac{e^{i k_{0} r}}{r} e^{-i \boldsymbol{k}^{\prime} \cdot \boldsymbol{r}^{\prime}}$ where $\boldsymbol{k}^{\prime}=k_{0} \boldsymbol{n}$. In that case,

$$
\psi(\boldsymbol{r}) \sim f\left(\boldsymbol{k}^{\prime}\right) \frac{e^{i k r}}{r} \text { where } f\left(\boldsymbol{k}^{\prime}\right)=\int_{\text {all space }} e^{-i \boldsymbol{k}^{\prime} \cdot \boldsymbol{r}^{\prime}} \sigma\left(\boldsymbol{r}^{\prime}\right) d V^{\prime} .
$$

This is analogous to the formalism we introduced in Section 11.4.2 in our discussion of spherical waves.

As one would expect, (12.3.15) and (12.3.16) both become the Green's function (12.2.25) for Poisson's equation in the limit as $k_{0} \rightarrow 0$.

\subsubsection{General Time Dependence}

If the source term $\sigma(\boldsymbol{r}, t)$ has a general time dependence, we cannot separate the space and time dependence so easily and we do have to worry about initial conditions. Let us start by re-stating the problem. It consists of solving

$$
\nabla^{2} \psi(\boldsymbol{r}, t)-\frac{1}{c^{2}} \frac{\partial^{2} \psi(\boldsymbol{r}, t)}{\partial t^{2}}=\sigma(\boldsymbol{r}, t)
$$

where the source term $\sigma(\boldsymbol{r}, t)$ has a general time dependence and the solution $\psi(\boldsymbol{r}, t)$ is subject to

$$
\psi(\boldsymbol{r}, t) \text { or } \boldsymbol{n} \cdot \nabla \psi(\boldsymbol{r}, t) \text { given for } \boldsymbol{r} \text { on } S
$$

plus initial conditions

$$
\psi(\boldsymbol{r}, t) \text { and } \frac{\partial \psi(\boldsymbol{r}, t)}{\partial t} \text { given at an initial time } t=\tau \text { throughout } V \text {. }
$$


To tackle this we need the Green's function $G\left(\boldsymbol{r}, t ; \boldsymbol{r}^{\prime}, t^{\prime}\right)$ which is the solution of

$$
\nabla^{2} G\left(\boldsymbol{r}, t ; \boldsymbol{r}^{\prime}, t^{\prime}\right)-\frac{1}{c^{2}} \frac{\partial^{2}}{\partial t^{2}} G\left(\boldsymbol{r}, t ; \boldsymbol{r}^{\prime}, t^{\prime}\right)=\delta\left(\boldsymbol{r}-\boldsymbol{r}^{\prime}\right) \delta\left(t-t^{\prime}\right)
$$

that satisfies the boundary condition $G=0$ or $\boldsymbol{n} \cdot \nabla G=0$ for $\boldsymbol{r}$ on $S$ plus the initial condition $G\left(\boldsymbol{r}, t ; \boldsymbol{r}^{\prime}, t^{\prime}\right)=0$ for $t<t^{\prime}$. The latter condition follows from the principle of causality: $\mathrm{G}$ is a response to a stimulus at $t=t^{\prime}$ and so should be zero prior to that time. With the usual Green's theorem manipulations, one can show that $G\left(\boldsymbol{r}, t ; \boldsymbol{r}^{\prime}, t^{\prime}\right)=G\left(\boldsymbol{r}^{\prime},-t^{\prime} ; \boldsymbol{r},-t\right)$ and that the solution to our original problem is

$$
\begin{aligned}
\psi(\boldsymbol{r}, t) & =\int_{\tau}^{t} \int_{V} G\left(\boldsymbol{r}, t ; \boldsymbol{r}^{\prime}, t^{\prime}\right) \sigma\left(\boldsymbol{r}^{\prime}, t^{\prime}\right) d V^{\prime} d t^{\prime}-\int_{\tau}^{t} \int_{S}\left[G\left(\boldsymbol{r}, t ; \boldsymbol{r}^{\prime}, t^{\prime}\right) \boldsymbol{n} \cdot \nabla^{\prime} \psi\left(\boldsymbol{r}^{\prime}, t^{\prime}\right)\right. \\
& \left.-\psi\left(\boldsymbol{r}^{\prime}, t^{\prime}\right) \boldsymbol{n} \cdot \nabla^{\prime} G\left(\boldsymbol{r}, t ; \boldsymbol{r}^{\prime}, t^{\prime}\right) d S^{\prime} d t^{\prime}\right] \\
& -\frac{1}{c^{2}} \int_{V}\left[\left.G\left(\boldsymbol{r}, t ; \boldsymbol{r}^{\prime}, T\right) \frac{\partial}{\partial t^{\prime}} \psi\left(\boldsymbol{r}^{\prime}, t^{\prime}\right)\right|_{t^{\prime}=\tau}-\left.\psi\left(\boldsymbol{r}^{\prime}, T\right) \frac{\partial}{\partial t^{\prime}} G\left(\boldsymbol{r}, t ; \boldsymbol{r}^{\prime}, t^{\prime}\right)\right|_{t^{\prime}=\tau}\right] d V^{\prime}
\end{aligned}
$$

An analogous approach applies to the diffusion equation. To solve

$$
\nabla^{2} \psi(\boldsymbol{r}, t)-\frac{1}{\kappa} \frac{\partial \psi(\boldsymbol{r}, t)}{\partial t}=\sigma(\boldsymbol{r}, t)
$$

with $\psi(\boldsymbol{r}, t)$ or $\boldsymbol{n} \cdot \nabla \psi(\boldsymbol{r}, t)$ given for $\boldsymbol{r}$ on $S$ plus the initial condition $\psi(\boldsymbol{r}, t)$ given at $t=\tau$, we first determine the Green's function $G\left(\boldsymbol{r}, t ; \boldsymbol{r}^{\prime}, t^{\prime}\right)$ which is the solution of

$$
\nabla^{2} G\left(\boldsymbol{r}, t ; \boldsymbol{r}^{\prime}, t^{\prime}\right)-\frac{1}{\kappa} \frac{\partial}{\partial t} G\left(\boldsymbol{r}, t ; \boldsymbol{r}^{\prime}, t^{\prime}\right)=\delta\left(\boldsymbol{r}-\boldsymbol{r}^{\prime}\right) \delta\left(t-t^{\prime}\right)
$$

that satisfies the boundary condition

$$
G\left(\boldsymbol{r}, t ; \boldsymbol{r}^{\prime}, t^{\prime}\right)=0 \text { or } \boldsymbol{n} \cdot \nabla G=0 \text { for } \boldsymbol{r} \text { on } S
$$

plus the initial condition $G\left(\boldsymbol{r}, t ; \boldsymbol{r}^{\prime}, t^{\prime}\right)=0$ for $t<t^{\prime}$.

One can again show that $G\left(\boldsymbol{r}, t ; \boldsymbol{r}^{\prime}, t^{\prime}\right)=G\left(\boldsymbol{r}^{\prime},-t^{\prime} ; \boldsymbol{r},-t\right)$ and

$$
\begin{aligned}
\psi(\boldsymbol{r}, t)= & \int_{\tau}^{t} \int_{V} G\left(\boldsymbol{r}, t ; \boldsymbol{r}^{\prime}, t^{\prime}\right) \sigma\left(\boldsymbol{r}^{\prime}, t^{\prime}\right) d V^{\prime} d t^{\prime} \\
& -\int_{\tau}^{t} \int_{S}\left[G\left(\boldsymbol{r}, t ; \boldsymbol{r}^{\prime}, t^{\prime}\right) \boldsymbol{n} \cdot \nabla^{\prime} \psi\left(\boldsymbol{r}^{\prime}, t^{\prime}\right)-\psi\left(\boldsymbol{r}^{\prime}, t^{\prime}\right) \boldsymbol{n} \cdot \nabla^{\prime} G\left(\boldsymbol{r}, t ; \boldsymbol{r}^{\prime}, t^{\prime}\right)\right] d S^{\prime} d t^{\prime} \\
& -\frac{1}{\kappa} \int_{V} G\left(\boldsymbol{r}, t ; \boldsymbol{r}^{\prime}, \tau\right) \psi\left(\boldsymbol{r}^{\prime}, \tau\right) d V^{\prime} .
\end{aligned}
$$




\subsubsection{The Wave and Diffusion Equation Green's Functions for Boundaries at Infinity}

The Green's function associated with the wave equation PDE and Dirichlet boundary conditions at infinity is a solution of

$$
\left(\nabla^{2}-\frac{1}{c^{2}} \frac{\partial^{2}}{\partial t^{2}}\right) G\left(\boldsymbol{r}, t ; \boldsymbol{r}^{\prime}, t^{\prime}\right)=\delta\left(\boldsymbol{r}-\boldsymbol{r}^{\prime}\right) \delta\left(t-t^{\prime}\right)
$$

that is everywhere bounded as a function of both $\boldsymbol{r}$ and $t$. The solution by means of Fourier transforms proceeds exactly as in Section 12.3.3 and yields

$$
G\left(\boldsymbol{r}, t ; \boldsymbol{r}^{\prime}, t^{\prime}\right)=-\frac{c^{2}}{(2 \pi)^{4}} \int_{-\infty}^{\infty} \int_{\text {all }} e^{-i \boldsymbol{k} \cdot\left(\boldsymbol{r}-\boldsymbol{r}^{\prime}\right)} \frac{e^{-i \omega\left(t-t^{\prime}\right)}}{c^{2} k^{2}-\omega^{2}} k^{2} d k \sin \theta_{k} d \theta_{k} d \varphi_{k} d \omega .
$$

As we saw in Chapter 3, the integral

$$
\Delta \equiv \int_{-\infty}^{\infty} \frac{e^{-i \omega\left(t-t^{\prime}\right)}}{c^{2} k^{2}-\omega^{2}} d \omega
$$

has four different values depending on how one avoids the poles at $\omega= \pm c k$. However, only one of these four satisfies our (causal) initial condition $G\left(\boldsymbol{r}, t ; \boldsymbol{r}^{\prime}, t^{\prime}\right)=0$ for $t<t^{\prime}$. This is the so-called retarded solution

$$
\Delta_{r e t}=\left\{\begin{array}{cl}
\frac{2 \pi \sin c k\left(t-t^{\prime}\right)}{c k} & \text { for } t>t^{\prime} \\
0 & \text { for } t<t^{\prime}
\end{array}\right.
$$

which arises from deforming the contour of integration to pass above both poles. The name is a reflection of the correspondence to a signal emitted at a time $t^{\prime}$ that is earlier or retarded compared to the time of arrival $t$.

Inserting (12.3.29) into (12.3.27) yields the (retarded) Green's function

$$
G\left(\boldsymbol{r}, t ; \boldsymbol{r}^{\prime}, t^{\prime}\right)=\left\{\begin{array}{c}
-\frac{c}{2 \pi} \int_{\text {all }} e_{\text {space }} e^{-i \boldsymbol{k} \cdot\left(\boldsymbol{r}-\boldsymbol{r}^{\prime}\right)} \frac{\sin c k\left(t-t^{\prime}\right)}{k} k^{2} d k d\left(\cos \theta_{k}\right) d \varphi_{k} \\
0
\end{array}\right.
$$

for $t>t^{\prime}$ and $t<t^{\prime}$, respectively. Choosing the $k_{3}$ axis to be in the direction of $\boldsymbol{r}-\boldsymbol{r}^{\prime}$, the angular integration is easy to perform and gives us

$$
\int_{\text {all space }} e^{-i \boldsymbol{k} \cdot\left(\boldsymbol{r}-\boldsymbol{r}^{\prime}\right)} \frac{\sin c k\left(t-t^{\prime}\right)}{k} k^{2} d k d\left(\cos \theta_{k}\right) d \varphi_{k}=\frac{4 \pi}{R} \int_{0}^{\infty} \sin k R \sin c k T d k
$$


where $R=\left|\boldsymbol{r}-\boldsymbol{r}^{\prime}\right|$ and $T=t-t^{\prime}$. Converting the sines on the right hand side into exponentials, we obtain

$$
\begin{aligned}
\int_{0}^{\infty} \sin k R \sin c k T d k & =-\frac{1}{4} \int_{0}^{\infty}\left[e^{i k(R+c T)}+e^{-i k(R+c T)}-e^{i k(R-c T)}-e^{-i k(R-c T)}\right] d k \\
& =-\frac{1}{4} \int_{-\infty}^{\infty}\left[e^{i k(R+c T)}-e^{i k(R-c T)}\right] d k \\
& =\frac{1}{4}[\delta(R-c T)-\delta(R+c T)] .
\end{aligned}
$$

Since the second delta function does not contribute for $T>0$ and since

$$
\delta(R-c T)=\frac{1}{c} \delta\left(\frac{R}{c}-T\right),
$$

substitution back into (12.3.30) gives us

$$
G\left(\boldsymbol{r}, t ; \boldsymbol{r}^{\prime}, t^{\prime}\right)=\left\{\begin{array}{cc}
-\frac{1}{4 \pi} \delta\left(\frac{\left|\boldsymbol{r}-\boldsymbol{r}^{\prime}\right|}{c}-\left(t-t^{\prime}\right)\right) & \text { for } t>t^{\prime} \\
0 & \text { for } t<t^{\prime}
\end{array}\right.
$$

as our final result for the (retarded) Green's function.

Given the unusual appearance of this Green's function, it is helpful to keep in mind its physical interpretation. It is a spherical wave produced by an instantaneous disturbance at the single point $\boldsymbol{r}=\boldsymbol{r}^{\prime}$ and time $t=t^{\prime}$. Prior to $t=t^{\prime}$, at times $t<t^{\prime}$, there is no wave. After $t=t^{\prime}$, at times $t>t^{\prime}$, the wave spreads out (propagates) to the location $\left|\boldsymbol{r}-\boldsymbol{r}^{\prime}\right|=c\left(t-t^{\prime}\right)$ and as it spreads, its amplitude decreases as $\frac{1}{\left|\boldsymbol{r}-\boldsymbol{r}^{\prime}\right|}$. A close analogue is the wave produced by dropping a small but massive pebble into a quiet pool of water.

Equation (12.3.22) is the prescription for superposing all of these elementary waves, part originating from the continuous source described by the function $\sigma(\boldsymbol{r}, t)$ and part from the boundary and initial conditions. Maximal simplification is obtained for the case of boundaries at infinity, an initial time $\tau \rightarrow-\infty$, and the requirement that $\psi(\boldsymbol{r}, t) \rightarrow 0$ as $r \rightarrow \infty$ and $t \rightarrow-\infty$. What results is a single integral of the form

$$
\psi(\boldsymbol{r}, t)=-\frac{1}{4 \pi} \int_{\text {all }} \frac{\sigma\left(\boldsymbol{r}^{\prime}-\frac{\left|t-t^{\prime}\right|}{c}\right)}{\left|\boldsymbol{r}-\boldsymbol{r}^{\prime}\right|} d V^{\prime}
$$

which is referred to in electrodynamics as the "retarded potential" solution of the wave equation.

Let us now turn our attention to the diffusion equation. Using Fourier transforms again to solve

$$
\nabla^{2} G\left(\boldsymbol{r}, t ; \boldsymbol{r}^{\prime}, t^{\prime}\right)-\frac{1}{\kappa} \frac{\partial}{\partial t} G\left(\boldsymbol{r}, t ; \boldsymbol{r}^{\prime}, t^{\prime}\right)=\delta\left(\boldsymbol{r}-\boldsymbol{r}^{\prime}\right) \delta\left(t-t^{\prime}\right),
$$


we find

$$
G\left(\boldsymbol{r}, t ; \boldsymbol{r}^{\prime}, t^{\prime}\right)=-\frac{i \kappa}{(2 \pi)^{4}} \int_{-\infty}^{\infty} \int_{\text {all }} \frac{e^{-i \boldsymbol{k} \cdot\left(\boldsymbol{r}-\boldsymbol{r}^{\prime}\right)-i \omega\left(t-t^{\prime}\right)}}{\omega+i \kappa k^{2}} k^{2} d k d\left(\cos \theta_{k}\right) d \varphi_{k} d \omega .
$$

The integral over $\omega$ can be evaluated by a simple application of the residue calculus. The integrand has a single simple pole located at $\omega=-i \kappa k^{2}$ in the lower half of the complex $\omega$ plane. If $t<t^{\prime}$, the contour will have to be closed in the upper half plane excluding the pole and resulting in a null result. But, for $t>t^{\prime}$, we must close the contour in the lower half plane and thus pick up a non-zero contribution from its residue. Thus,

$$
G\left(\boldsymbol{r}, t ; \boldsymbol{r}^{\prime}, t^{\prime}\right)=\left\{\begin{array}{c}
-\frac{\kappa}{(2 \pi)^{3}} \int_{\text {all }} \int_{\text {space }} e^{-i \boldsymbol{k} \cdot\left(\boldsymbol{r}-\boldsymbol{r}^{\prime}\right)-\kappa k^{2}\left(t-t^{\prime}\right)} k^{2} d k d\left(\cos \theta_{k}\right) d \varphi_{k} \\
0
\end{array}\right.
$$

for $t>t^{\prime}$ and $t<t^{\prime}$, respectively. This result inherently meets the initial condition that we wanted to impose on this Green's function.

The integral is a three-dimensional version of one we evaluated in Chapter 3. If we set $\boldsymbol{R}=\boldsymbol{r}-\boldsymbol{r}^{\prime}$ and $T=t-t^{\prime}$ and complete the square in the exponent, it becomes

$$
\begin{aligned}
& \int_{\text {all space }} e^{-i \boldsymbol{k} \cdot\left(\boldsymbol{r}-\boldsymbol{r}^{\prime}\right)-\kappa k^{2}\left(t-t^{\prime}\right)} k^{2} d k d\left(\cos \theta_{k}\right) d \varphi_{k}=e^{-\frac{R^{2}}{4 k T}} \int_{\text {all space }} e^{-\kappa T\left(k-i \frac{R}{2 k T}\right)^{2}} d k_{1} d k_{2} d k_{3} \\
& =e^{-\frac{R^{2}}{4 k T}}\left(\int_{-\infty}^{\infty} e^{-\kappa T\left(k_{1}-i \frac{X}{2 \kappa T}\right)^{2}} d k_{1}\right)^{3}
\end{aligned}
$$

where we have switched from spherical to rectangular coordinates and set $x-x^{\prime}=$ $X$. The integral in the second line was evaluated in Chapter 3 and we found that it equals $\sqrt{\frac{\pi}{\kappa T}}$. Therefore, substituting all this information back into (12.3.33), we obtain as our final expression

$$
G\left(\boldsymbol{r}, t ; \boldsymbol{r}^{\prime}, t^{\prime}\right)=\left\{\begin{array}{cl}
-\frac{\kappa}{\left[4 \pi \kappa\left(t-t^{\prime}\right)\right]^{3 / 2}} \exp \left[-\left|\boldsymbol{r}-\boldsymbol{r}^{\prime}\right|^{2} / 4 \kappa\left(t-t^{\prime}\right)\right] & \text { for } t>t^{\prime} \\
0 & \text { for } t<t^{\prime}
\end{array}\right.
$$

This is a sharply peaked (Gaussian) function of $\left|\boldsymbol{r}-\boldsymbol{r}^{\prime}\right|$ for small values of $t-t^{\prime}$ and broad for large values of $t-t^{\prime}$. In fact, in the limit as $t \rightarrow t^{\prime}$, the Green's function $G\left(\boldsymbol{r}, t ; \boldsymbol{r}^{\prime}, t^{\prime}\right) \rightarrow-\kappa \delta\left(\boldsymbol{r}-\boldsymbol{r}^{\prime}\right)$. Thus, it describes how a delta function impulse at $t=t^{\prime}$ diffuses through the medium at later times.

We can now use (12.3.25) to write down the solution to a diffusion problem involving a source $\sigma(\boldsymbol{r}, t)$, boundary condition $\psi \rightarrow 0$ (and $G \rightarrow 0$ ) as $\left|\boldsymbol{r}-\boldsymbol{r}^{\prime}\right| \rightarrow \infty$, and initial condition $\psi(\boldsymbol{r}, t)$ given at $t=\tau$ : it is 


$$
\begin{aligned}
\psi(\boldsymbol{r}, t)= & \frac{1}{\sqrt{4 \pi \kappa}} \int_{\tau}^{t} \int_{\text {all space }} \frac{1}{\left(t-t^{\prime}\right)^{3 / 2}} \exp \left[-\left|\boldsymbol{r}-\boldsymbol{r}^{\prime}\right|^{2} / 4 \kappa\left(t-t^{\prime}\right)\right] \sigma\left(r^{\prime}, t^{\prime}\right) d t^{\prime} d V^{\prime} \\
& +\frac{1}{[4 \pi \kappa(t-\tau)]^{3 / 2}} \int_{\text {all space }} \exp \left[-\left|\boldsymbol{r}-\boldsymbol{r}^{\prime}\right|^{2} / 4 \kappa(t-\tau)\right] \psi\left(r^{\prime}, \tau\right) d V^{\prime}
\end{aligned}
$$

In particular, if the source $\sigma(\boldsymbol{r}, t) \equiv 0$ and $\psi(\boldsymbol{r}, 0)=\delta(x-a)$, the solution is

$$
\psi(\boldsymbol{r}, t)=\psi(x, t)=\frac{1}{\sqrt{4 \pi \kappa t}} e^{-\frac{(x-a)^{2}}{4 k t}}
$$

which describes the diffusion in both $\mathrm{x}$-directions of a substance initially confined in the yz-plane at $x=a$. 